\title{
Dual Affine invariant points *
}

\author{
Mathieu Meyer, Carsten Schütt and Elisabeth M. Werner ${ }^{\dagger}$
}

\begin{abstract}
An affine invariant point on the class of convex bodies $\mathcal{K}_{n}$ in $R^{n}$, endowed with the Hausdorff metric, is a continuous map from $\mathcal{K}_{n}$ to $\mathbb{R}^{n}$ which is invariant under one-to-one affine transformations $A$ on $\mathbb{R}^{n}$, that is, $p(A(K))=A(p(K))$.

We define here the new notion of dual affine point $q$ of an affine invariant point $p$ by the formula $q\left(K^{p(K)}\right)=p(K)$ for every $K \in \mathcal{K}_{n}$, where $K^{p(K)}$ denotes the polar of $K$ with respect to $p(K)$.

We investigate which affine invariant points do have a dual point, whether this dual point is unique and has itself a dual point. We define a product on the set of affine invariant points, in relation with duality.

Finally, examples are given which exhibit the rich structure of the set of affine invariant points.
\end{abstract}

\footnotetext{
${ }^{*}$ Keywords: affine invariant point, dual affine invariant point. 2010 Mathematics Subject Classification: 52A20, 53A 15

${ }^{\dagger}$ Partially supported by an NSF grant
} 


\section{Introduction.}

While convex bodies have been the topic of extensive research for more than a century, it is the affine geometry of these bodies that has been a main focus of study in recent years. We only mention the rapid progress in the $L_{p}$ Brunn Minkowski theory (e.g., [2, 4, 8, 10, 21, 22, 26, 27]) and the theory of valuations e.g., [5, 6, 7, 19]. The resulting body of work has proved to be a valuable tool in fields such as harmonic analysis, information theory, stochastic geometry and PDEs (e.g., [9, 14, 15, 25]).

Specific points associated to a convex body, like the centroid and the Santaló point, satisfy an affine invariance property: The point of an affine image of a convex body is the affine image of the point. More formally, if $\mathcal{K}_{n}$ denotes the set of all convex bodies in $\mathbb{R}^{n}$, a mapping $p: \mathcal{K}_{n} \rightarrow \mathbb{R}^{n}$ is an affine invariant point if $p$ is continuous for the Hausdorff topology and satisfies

$$
p(T(K))=T(p(K))
$$

for every one-to-one affine mapping $T: \mathbb{R}^{n} \rightarrow \mathbb{R}^{n}$.

Even though this notion is intriguing in its simplicity, little is known about affine invariant points. At the same time, these are fundamental invariants of convex sets. They are, for instance, useful to characterize properties of symmetry or of non symmetry of convex bodies (e.g., [12 and [13). The more different affine invariant points a convex body has the less symmetric it is. The task of computing an affine invariant point of a convex body can be formidable, even to show that two affine invariant points of a convex body are different can be nontrivial.

Affine invariant points were first defined by B. Grünbaum in 1963 in his seminal paper 3, where he also posed several open problems. In two preceding papers, 12 and 13, we answered some of Grünbaum's questions: The dimension of the space of affine invariant points is infinte and there are convex bodies $K$ in $\mathbb{R}^{n}$ such that every point in $\mathbb{R}^{n}$ is an affine invariant point of $K$. More importantly, we showed in some cases that the presence of many affine invariant points means that the convex body lacks symmetry.

However, many structural questions are still open. In this paper we address them through the study of duality. We introduce the new notion of dual affine invariant point. In short, the point $q$ is a dual affine invariant point to $p$ if

$$
q\left(K^{p(K)}\right)=p(K)
$$

for all convex bodies $K$. Here, $K^{z}=\left\{y \in \mathbb{R}^{n}:\langle y-z, x-z)\right\rangle \leq 1$ for every $\left.x \in K\right\}$ is the polar of $K$ with respect to the point $z$. The motivation for our definition, given in Section 3 comes from the duality relation between the center of gravity and the Santaló point of a convex body. Further examples of dual affine invariant points are the center of the John ellipsoid and the center of the Löwner ellipsoid. All this is explained in Section 5.

We start our study by addressing a number of basic questions. First, does every affine invariant point $p$ have a dual $p^{\circ}$ ? The answer, surprisingly, is: No. This is the content of Theorem 2, In Theorem 1, we show that if a dual affine invariant point exists, it is unique. Theorem 3 establishes a reflexivity principle for affine invariant points, namely that the double dual $p^{\circ \circ}$ of $p$ equals $p$. The proofs of the theorems require a number of technical results. Those are presented in Section 3 
In Section 4 we give the proof of the main theorems. We also define there a product $[p, q]$ of two affine invariant points $p$ and $q$ as a mapping from from the set $\mathcal{P}_{n}$ of all affine invariant points into itself. This product has a nice duality property,

$$
\left[q^{\circ}, p^{\circ}\right] \circ[p, q]=I_{n},
$$

where $I_{n}$ is the identity on $\mathcal{P}_{n}$.

Finally, Section 5 is devoted to a list of useful examples of affine invariant points and a related notion, that of affine invariant sets (also defined in Section 2), many new ones among them. Moreover, we investigate how to extend the affine invariant points on $\mathcal{K}_{n, k}$, the set of compact convex subsets in $\mathbb{R}^{n}$ whose affine span is $k$-dimensional, to affine invariant points on $\mathcal{K}_{n}$.

The authors would like to thank the American Institute of Mathematics in Palo Alto where, in the course of the workshop "Invariants in convex geometry and Banach space theory", much of the paper was produced.

\section{Notation and Background material.}

We denote by $\mathcal{K}_{n}$ the set of all convex bodies in $\mathbb{R}^{n}$, that is the set of all convex compact sets with nonempty interior. For $K \in \mathcal{K}_{n}, \operatorname{int}(K)$ is the interior of $K$ and $\partial K$ is its boundary. We say that $K \in \mathcal{K}_{n}$ is in $C_{+}^{2}$, if $\partial K$ is $C^{2}$ with strictly positive Gaussian curvature.

The Euclidean ball centered at $a$ with radius $r$ is $B_{2}^{n}(a, r)$. We write in short $B_{2}^{n}=$ $B_{2}^{n}(0,1)$ and $S^{n-1}=\partial B_{2}^{n}$. We endow $\mathbb{R}^{n}$ with its canonical scalar product, and for $x \in \mathbb{R}^{n}$, we denote $|x|=\sqrt{\langle x, x\rangle}$ its Euclidean norm. The $n$-dimensional volume of $K$ is $\operatorname{vol}_{n}(K)$, or simply $|K|$. Quite often, if $A \subset \mathbb{R}^{n}$ has an affine span of dimension $k$, we shall denote also by $|A|$ the $k$-dimensional volume of $A$ in its affine span.

For subsets $A$ and $B$ of $\mathbb{R}^{n}$, $\operatorname{conv}[A, B]$ denotes their convex hull, the smallest convex body containing them.

The support function $h_{K}: \mathbb{R}^{n} \rightarrow \mathbb{R}$ of a convex body $K$ is given by $h_{K}(\xi)=$ $\sup _{x \in K}\langle\xi, x\rangle$. If $0 \in \operatorname{int}(K), K^{\circ}=\left\{y \in \mathbb{R}^{n}:\langle x, y\rangle \leq 1\right\}$ is the polar body of $K$ with respect to 0 . More generally, we define the polar body $K^{x}$ of $K$ with respect to $x \in \mathbb{R}^{n}$ by

$$
K^{x}=(K-x)^{\circ}+x, \text { or } K^{x}-x=(K-x)^{\circ} .
$$

Note that $K^{x} \in \mathcal{K}_{n}$ if and only if $x \in \operatorname{int}(K)$. We will only consider such situations. By the bipolar theorem,

$$
\left(K^{x}\right)^{x}=K,
$$

which may be written as

$$
K-x=\left(K^{x}-x\right)^{\circ} .
$$

We shall frequently use the fact that if $T: \mathbb{R}^{n} \rightarrow \mathbb{R}^{n}$ is a one-to-one linear map, $K \in \mathcal{K}_{n}$ and $x \in \operatorname{int}(K)$, then

$$
(T(K-x))^{\circ}=T^{*-1}\left((K-x)^{\circ}\right) .
$$


Here $T^{*}$ is the adjoint of $T$ and $T^{*-1}$ its inverse.

The Hausdorff metric $d_{H}$ on $\mathcal{K}_{n}$ is defined as

$$
d_{H}\left(K_{1}, K_{2}\right)=\min \left\{\lambda \geq 0: K_{1} \subseteq K_{2}+\lambda B_{2}^{n}, K_{2} \subseteq K_{1}+\lambda B_{2}^{n}\right\} .
$$

Now we recall the definitions of affine invariant points and of affine invariant sets [3, 13].

Definition 1. A map $p: \mathcal{K}_{n} \rightarrow \mathbb{R}^{n}$ is called affine invariant point, if $p$ is continuous and if for every nonsingular affine map $T: \mathbb{R}^{n} \rightarrow \mathbb{R}^{n}$, one has

$$
p(T(K))=T(p(K)) .
$$

We denote by $\mathfrak{P}_{n}$ the set of affine invariant points in $\mathbb{R}^{n}$,

$$
\mathfrak{P}_{n}=\left\{p: \mathcal{K}_{n} \rightarrow \mathbb{R}^{n} \mid p \text { is continuous and affine invariant }\right\},
$$

and for a fixed $K \in \mathcal{K}_{n}, \mathfrak{P}_{n}(K)=\left\{p(K) ; p \in \mathfrak{P}_{n}\right\}$.

We say that $p \in \mathfrak{P}_{n}$ is proper if for all $K \in \mathcal{K}_{n}$, one has $p(K) \in \operatorname{int}(K)$.

Definition 2. A map $A: \mathcal{K}_{n} \rightarrow \mathcal{K}_{n}$ is an affine invariant set mapping, or an affine invariant set, if $A$ is continuous (when $\mathcal{K}_{n}$ is endowed with the Hausdorff metric) and if for every affine one-to-one map $T: \mathbb{R}^{n} \rightarrow \mathbb{R}^{n}$, one has

$$
A(T(K))=T(A(K)) .
$$

We denote by $\mathfrak{A}_{n}$ the set of all affine invariant set mappings from $\mathcal{K}_{n}$ to $\mathcal{K}_{n}$.

Well known classical examples (see e.g. [3, 13]) of proper affine invariant points of a convex body $K$ in $\mathbb{R}^{n}$ are the centroid,

$$
g(K)=\frac{\int_{K} x d x}{|K|}
$$

the Santaló point, which is the unique point $s(K) \in \operatorname{int}(K)$ for which $\left|K^{s(K)}\right|=\min _{x}\left|K^{x}\right|$, the center of the John ellipsoid of $K$, that is the ellipsoid of maximal volume contained in $K$ and the center of the Löwner ellipsoid of $K$, that is the ellipsoid of minimal volume containing $K$.

We will discuss these and other examples in Section 5. More details on affine invariant points, and some results that we shall use here, can be found in [13.

\section{Dual affine invariant points.}

We now introduce the new concept of a dual of an affine invariant point.

Definition 3. Let $p \in \mathfrak{P}_{n}$ be proper. 
(i) We say $q \in \mathfrak{P}_{n}$ is a dual of $p$ if for all $K \in \mathcal{K}_{n}$

$$
q\left(K^{p(K)}\right)=p(K)
$$

If $p$ has a unique dual, we denote it by $p^{\circ}$.

(ii) Let $w \in \mathfrak{P}_{n}$. We say that $w$ is a bidual of $p$, if $p$ has a proper dual $q \in \mathfrak{P}_{n}$, such that $w$ is a dual of $q$. This means that there is an affine invariant point $q$ that is dual to $p$ and that $w$ is dual to $q$,

$$
q\left(K^{p(K)}\right)=p(K) \quad \text { and } \quad w\left(K^{q(K)}\right)=q(K) .
$$

Theorem 3 assures that a dual point is automatically proper.

The centroid and the Santaló point, and the center of the John ellipsoid of $K$ and the center of the Löwner ellipsoid are examples of dual affine invariant points. We will explain this in Section 5 .

For $q \in \mathfrak{P}_{n}$, the affine invariance of the mapping $K \rightarrow q\left(K^{p(K)}\right)$ implies that $q$ is a dual of $p$. This is the content of the following lemma.

Lemma 1. Let $p, q \in \mathfrak{P}_{n}$. Suppose that $p$ is proper. Then $q$ is a dual of $p$ if and only if the mapping $r: \mathcal{K}_{n} \rightarrow \mathbb{R}^{n}$ defined by $r(K)=q\left(K^{p(K)}\right)$ is itself an affine invariant point.

Proof. By definition, if $q$ is the dual of $p$, then $r=p \in \mathfrak{P}_{n}$. Conversely, suppose that the map $K \rightarrow q\left(K^{p(K)}\right)$ is an affine invariant point. For all $K \in \mathcal{K}_{n}$, all linear, invertible maps $T: \mathbb{R}^{n} \rightarrow \mathbb{R}^{n}$ and all $b \in \mathbb{R}^{n}$ we have by (2)

$$
(T(K)+b)^{p(T(K)+b)}-p(T(K)+b)=(T(K-p(K)))^{\circ}=T^{*-1}\left((K-p(K))^{\circ}\right) .
$$

Therefore, and as $q$ and $r$ are affine invariant points,

$$
\begin{aligned}
T^{*-1}\left(q\left((K-p(K))^{\circ}\right)\right) & =q\left(T^{*-1}\left((K-p(K))^{\circ}\right)\right) \\
& =q\left((T(K)+p(K))^{p(T(K)+p(K))}-p(T(K)+p(K))\right) \\
& =q\left((T(K)+p(K))^{p(T(K)+p(K))}\right)-p(T(K)+p(K)) \\
& =r(T(K)+p(K))-p(T(K)+p(K))=T(r(K)-p(K)) \\
& =T\left(q\left((K-p(K))^{\circ}\right)\right) .
\end{aligned}
$$

In particular, $T^{*-1}\left(q\left((K-p(K))^{\circ}\right)\right)=T\left(q\left((K-p(K))^{\circ}\right)\right)$ holds for $T=\lambda I d$ with $\lambda>1$. One has thus $q\left((K-p(K))^{\circ}\right)=0$ for all $K$, and hence $r(K)=p(K)$ for all $K \in \mathcal{K}_{n}$.

We will show in Theorem 3 that if a proper affine invariant point $p$ has a dual, then this dual point is unique and proper. We will then show that $p$ is the unique dual of $p^{\circ}$, and hence $p$ has a unique bidual point which is $p$ itself.

First, we give a definition which will be useful to investigate duality. 
Definition 4. Let $p \in \mathfrak{P}_{n}$ be proper .

We say that $p$ is injective if, whenever $K_{1}, K_{2} \in \mathcal{K}_{n}$ satisfy $K_{1}^{p\left(K_{1}\right)}=K_{2}^{p\left(K_{2}\right)}$, then $p\left(K_{1}\right)=p\left(K_{2}\right)$.

We say that $p$ is surjective if for every $C \in \mathcal{K}_{n}$, there exists $K \in \mathcal{K}_{n}$ such that $C=K^{p(K)}$.

We say that $p$ is bijective if it is both injective and surjective.

The centroid, the Santaló point the center of the John ellipsoid and the center of the Löwner ellipsoid are examples of injective and surjective affine invariant points. More examples are given in Section 5 .

Remark 1. Let $p \in \mathfrak{P}_{n}$ be proper and define $\phi_{p}: \mathcal{K}_{n} \rightarrow \mathcal{K}_{n}$ by

$$
\phi_{p}(K)=K^{p(K)} .
$$

It is easy to see that $\phi_{p}$ is continuous. Moreover we have

(i) $p$ is injective, (surjective, bijective) iff $\phi_{p}$ is injective, (surjective, bijective).

We address the first statement. Let $p$ be injective and suppose that $\phi_{p}\left(K_{1}\right)=\phi_{p}\left(K_{2}\right)$, i.e. $K_{1}^{p\left(K_{1}\right)}=K_{2}^{p\left(K_{2}\right)}$. Then, by injectivity of $p, K_{1}=K_{2}$, i.e. $\phi_{p}$ is injective. Conversely, let $\phi_{p}$ be injective and suppose that $K_{1}^{p\left(K_{1}\right)}=K_{2}^{p\left(K_{2}\right)}$. The latter means exactly that $\phi_{p}\left(K_{1}\right)=\phi_{p}\left(K_{2}\right)$, and it follows from the injectivity of $\phi_{p}$, that $K_{1}=K_{2}$.

(ii) $q \in \mathfrak{P}_{n}$ is dual of $p$ if and only if $q \circ \phi_{p}=p$.

The next two lemmas characterize injectivity and surjectivity.

Lemma 2. Let $p$ be a proper affine invariant point. The following are equivalent.

(i) $p$ is surjective.

(ii) For every $C$ in $\mathcal{K}_{n}$ there is a $z \in \operatorname{int}(C)$ such that $p\left((C-z)^{\circ}\right)=0$.

Proof. $p$ is surjective means that for all $C$ in $\mathcal{K}_{n}$ there is $K$ in $\mathcal{K}_{n}$ such that

$$
C=K^{p(K)}=(K-p(K))^{\circ}+p(K),
$$

or, equivalently, $(C-p(K))^{\circ}=K-p(K)$. This is equivalent to

$$
p\left((C-p(K))^{\circ}\right)=p(K)-p(K)=0 .
$$

Lemma 3. Let $p$ be a proper affine invariant point. Then the following are equivalent. (i) $p$ is injective.

(ii) For all $C \in \mathcal{K}_{n}$, there exists at most one $z \in \operatorname{int}(C)$ such that $p\left((C-z)^{\circ}\right)=0$.

Proof. $(i) \Rightarrow($ ii $)$ Suppose that there are $z_{1}$ and $z_{2} \operatorname{in} \operatorname{int}(C)$ such that $p\left(\left(C-z_{1}\right)^{\circ}\right)=$ $p\left(\left(C-z_{2}\right)^{\circ}\right)=0$. For $i=1,2$, we put $K_{i}=C^{z_{i}}=\left(C-z_{i}\right)^{\circ}+z_{i}$. Then $K_{i}-z_{i}=\left(C-z_{i}\right)^{\circ}$ and hence

$$
\left.0=p\left(\left(C-z_{i}\right)^{\circ}\right)=p\left(K_{i}-z_{i}\right)\right)=p\left(K_{i}\right)-z_{i},
$$


and thus $p\left(K_{i}\right)=z_{i}$. By (1), $C=K_{1}^{z_{1}}=K_{1}^{p\left(K_{1}\right)}$ and $C=K_{2}^{z_{2}}=K_{2}^{p\left(K_{2}\right)}$. Injectivity of $p$ implies that $z_{1}=z_{2}$.

(ii) $\Rightarrow(i)$ Suppose $C=K_{1}^{p\left(K_{1}\right)}=K_{2}^{p\left(K_{2}\right)}$ for $K_{1}, K_{2} \in \mathcal{K}_{n}$. Then, for $i=1,2, C-$ $p\left(K_{i}\right)=\left(\left(K_{i}-p\left(K_{i}\right)\right)^{\circ}\right.$, so that $\left(C-p\left(K_{i}\right)\right)^{\circ}=K_{i}-p\left(K_{i}\right)$. Hence $C-p\left(K_{i}\right)$ has a bounded polar, which means that $p\left(K_{i}\right) \in \operatorname{int}(C)$. It follows that for $i=1,2$,

$$
p\left(\left(C-p\left(K_{i}\right)\right)^{\circ}\right)=p\left(K_{i}-p\left(K_{i}\right)\right)=0,
$$

and hence by (ii) that $p\left(K_{1}\right)=p\left(K_{2}\right)$.

It will be useful to have a new description of $\left(K^{\circ}-z\right)^{\circ}$ when $0 \in \operatorname{int}(K)$ and $z \in$ $\operatorname{int}\left(K^{\circ}\right)$. Let thus $K \in \mathcal{K}_{n}$ be such that $0 \in \operatorname{int}(K)$. For $z \in \operatorname{int}\left(K^{\circ}\right)$, we put

$$
K_{z}=\left\{\frac{x}{1-\langle x, z\rangle}: x \in K\right\} \text {. }
$$

In Lemma 4 we show that $K_{z}=\left(K^{\circ}-z\right)^{\circ}$.

It is easy to show

$$
\left|K_{z}\right|=\int_{K} \frac{d x}{(1-\langle x, z\rangle)^{n+1}} \quad \text { and } \quad \lim _{z \rightarrow \partial K^{\circ}}\left|K_{z}\right|=+\infty .
$$

Moreover, when $\lambda \rightarrow 1,\left|K_{\lambda z_{0}}\right| \rightarrow+\infty$ uniformly in $z_{0} \in \partial K^{\circ}$.

For any ellipsoid $\mathcal{E}$ centered at 0 , and every $z$ such that $h_{\mathcal{E}}(z)<1, \mathcal{E}_{z}$ is an ellipsoid. For the Euclidean unit ball $B_{2}^{n},\left(B_{2}^{n}\right)_{z}$ is an ellipsoid with center $\frac{z}{1-|z|^{2}}$ and

$$
\left|\left(B_{2}^{n}\right)_{z}\right|=\frac{\left|B_{2}^{n}\right|}{\left(1-|z|^{2}\right)^{\frac{n+1}{2}}} .
$$

Remark 2. Therefore, a natural question to ask is whether ellipsoids are the unique bodies such that $K_{z}$ is centrally symmetric for any $z \in K^{\circ}$.

The next lemma relates $K_{z}$ to $\left(K^{\circ}-z\right)^{\circ}$.

Lemma 4. For all $K \in \mathcal{K}_{n}$ with $0 \in \operatorname{int}(K)$ and all $z \in \operatorname{int}\left(K^{\circ}\right)$,

$$
\left(K^{\circ}\right)^{z}-z=\left(K^{\circ}-z\right)^{\circ}=K_{z} .
$$

By Lemma 4, for all $z \in \operatorname{int}\left(K^{\circ}\right)$ and all $z^{\prime} \in \operatorname{int}\left(\left(K_{z}\right)^{\circ}\right)=\operatorname{int}\left(K^{\circ}-z\right)$

$$
\left(K_{z}\right)_{z^{\prime}}=\left(\left(K_{z}\right)^{\circ}-z^{\prime}\right)^{\circ}=\left(K^{\circ}-\left(z+z^{\prime}\right)\right)^{\circ}=K_{z+z^{\prime}} .
$$

Proof. The first equality follows from the definition. For the second one, observe that for $z \in \operatorname{int}\left(K^{\circ}\right)$,

$$
\begin{aligned}
\left(K^{\circ}-z\right)^{\circ} & =\left\{x^{\prime} \in \mathbb{R}^{n}:\left\langle x^{\prime}, y-z\right\rangle \leq 1 \text { for all } y \in K^{\circ}\right\} \\
& =\left\{x^{\prime} \in \mathbb{R}^{n}:\left\langle x^{\prime}, y\right\rangle \leq 1+\left\langle x^{\prime}, z\right\rangle \text { for all } y \in K^{\circ}\right\} .
\end{aligned}
$$

Since $0 \in \operatorname{int}\left(K^{\circ}\right)$, such an $x^{\prime}$ satisfies $1+\left\langle x^{\prime}, z\right\rangle>0$, so that

$$
\left(K^{\circ}-z\right)^{\circ}=\left\{x^{\prime} \in \mathbb{R}^{n}:\left\langle\frac{x^{\prime}}{1+\left\langle x^{\prime}, z\right\rangle}, y\right\rangle \leq 1 \text { for all } y \in K^{\circ}\right\} .
$$


Since $\left(K^{\circ}\right)^{\circ}=K$, one has thus

$$
\left(K^{\circ}-z\right)^{\circ}=\left\{x^{\prime} \in \mathbb{R}^{n}: \frac{x^{\prime}}{1+\left\langle x^{\prime}, z\right\rangle} \in K\right\} .
$$

Finally, observe that $x=\frac{x^{\prime}}{1+\left\langle x^{\prime}, z\right\rangle}$ if and only if $x^{\prime}=\frac{x}{1-\langle x, z\rangle}$.

Now we show that for a proper affine invariant point $p$ and for the centroid $g, p\left(\left(K^{\circ}-\right.\right.$ $\left.\left.\lambda x_{0}\right)^{\circ}\right)$ and $g\left(\left(K^{\circ}-\lambda x_{0}\right)^{\circ}\right)$ have an analogous behavior when $\lambda \rightarrow 1$. To do so, we need several technical lemmas.

Lemma 5. Let $K$ be a convex body containing 0 in its interior. Then there are constants $c>0$ and $0<\lambda_{0}<1$ such that for all $x_{0}^{*} \in \partial K^{\circ}$, all proper $p \in \mathfrak{P}_{n}$ and all $\lambda_{0}<\lambda<1$,

$$
\left|p\left(K_{\lambda x_{0}^{*}}\right)\right| \geq\left\langle p\left(K_{\lambda x_{0}^{*}}\right), \frac{x_{0}^{*}}{\left|x_{0}^{*}\right|}\right\rangle \geq \frac{c}{1-\lambda} .
$$

Proof. It is well known that for every $L \in \mathcal{K}_{n}$ one has that $L-g(L) \subset n(g(L)-L)$, and thus $L-(n+1) g(L) \subset-n L$. Hence, for every $v \in S^{n-1}$,

$$
\langle g(L), v\rangle \geq \frac{1}{n+1}\left(h_{L}(v)-n h_{L}(-v)\right) .
$$

Since $p$ is proper, it follows from Proposition 1 of 13 that for some $0 \leq \alpha<1$, one has $p(L)-g(L) \in \alpha(L-g(L))$. Therefore, for every $v \in S^{n-1}$,

$$
\langle p(L), v\rangle \geq(1-\alpha)\langle g(L), v\rangle-\alpha h_{L}(-v) \geq \frac{1-\alpha}{n+1} h_{L}(v)-\left(\frac{n(1-\alpha)}{n+1}+\alpha\right) h_{L}(-v) .
$$

By Lemma 4, for all $\lambda$ with $0<\lambda<1$

$$
K_{\lambda x_{0}^{*}}=\left\{\frac{x}{1-\lambda\left\langle x_{0}^{*}, x\right\rangle}: x \in K\right\} .
$$

Consequently,

$$
h_{K_{\lambda x_{0}^{*}}}\left(\frac{x_{0}^{*}}{\left|x_{0}^{*}\right|}\right)=\frac{1}{\left|x_{0}^{*}\right|} \sup _{x \in K} \frac{\left\langle x_{0}^{*}, x\right\rangle}{1-\lambda\left\langle x, x_{0}^{*}\right\rangle}=\frac{1}{\left|x_{0}^{*}\right|(1-\lambda)}
$$

and

$$
h_{K_{\lambda x_{0}^{*}}}\left(-\frac{x_{0}^{*}}{\left|x_{0}^{*}\right|}\right)=\frac{1}{\lambda\left|x_{0}^{*}\right|} \sup _{x \in K} \frac{-\lambda\left\langle x_{0}^{*}, x\right\rangle}{1-\lambda\left\langle x, x_{0}^{*}\right\rangle} \leq \frac{1}{\lambda\left|x_{0}^{*}\right|} .
$$

Together with (77) we get

$$
\left\langle p\left(K_{\lambda x_{0}^{*}}\right), \frac{x_{0}^{*}}{\left|x_{0}^{*}\right|}\right\rangle \geq \frac{1}{\left|x_{0}^{*}\right|}\left(\frac{1-\alpha}{(1-\lambda)(n+1)}-\frac{1}{\lambda}\left(\frac{n(1-\alpha)}{n+1}+\alpha\right)\right) .
$$

We choose

$$
c=\frac{1-\alpha}{2(n+1) \max _{x_{0}^{*} \in \partial K^{\circ}}\left|x_{0}^{*}\right|}
$$

and we obtain the result, choosing a big enough $\lambda_{0}$, for all $\lambda$ with $0<\lambda_{0} \leq \lambda<1$. 
Lemma 6. For all $K \in \mathcal{K}_{n}$ with a $C_{2}^{+}$-boundary there exists $c^{\prime}>0$ such that for all $\left(x_{0}^{*}, x_{0}\right) \in \partial K^{\circ} \times \partial K$ satisfying $\left\langle x_{0}^{*}, x_{0}\right\rangle=1$ and all $x \in K$

$$
\left|x-x_{0}\right|^{2} \leq c^{\prime}\left(1-\left\langle x_{0}^{*}, x\right\rangle\right) .
$$

Proof. By the $C_{2}^{+}$hypothesis, there exists an $R>0$ such that for all $\left(x_{0}^{*}, x_{0}\right) \in \partial K^{\circ} \times \partial K$ with $\left\langle x_{0}^{*}, x_{0}\right\rangle=1$,

$$
K \subset x_{0}-R \frac{x_{0}^{*}}{\left|x_{0}^{*}\right|}+R B_{2}^{n} .
$$

Hence, for all $x \in K,\left|x_{0}-x-R \frac{x_{0}^{*}}{\left|x_{0}^{*}\right|}\right|^{2} \leq R^{2}$, or,

$$
\left|x_{0}-x\right|^{2} \leq \frac{2 R}{\left|x_{0}^{*}\right|}\left\langle x_{0}^{*}, x_{0}-x\right\rangle=\frac{2 R}{\left|x_{0}^{*}\right|}\left(1-\left\langle x_{0}^{*}, x\right\rangle\right) .
$$

We take $c^{\prime}=\frac{2 R}{\min _{y^{*} \in \partial K^{\circ}}\left|y^{*}\right|}$.

Lemma 7. For all $K \in \mathcal{K}_{n}$ with a $C_{2}^{+}$-boundary and $0 \in \operatorname{int}(K)$ there exists $d>0$ such that for all $\left(x_{0}^{*}, x_{0}\right) \in \partial K^{\circ} \times \partial K$ with $\left\langle x_{0}^{*}, x_{0}\right\rangle=1$, for all $v \in S^{n-1}$ with $\left\langle v, x_{0}\right\rangle=0$, for all $0<\lambda<1$ and for all $y \in K_{\lambda x_{0}^{*}}$,

$$
\langle v, y\rangle \leq \frac{d}{2 \sqrt{\lambda(1-\lambda)}}
$$

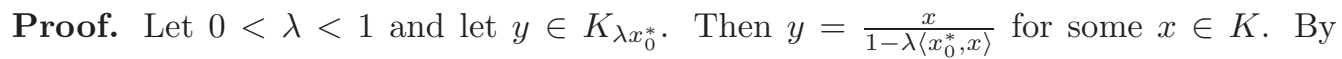
Lemma 6 and $\left\langle v, x_{0}\right\rangle=0$

$$
|\langle v, y\rangle|=\frac{|\langle x, v\rangle|}{1-\lambda\left\langle x_{0}^{*}, x\right\rangle} \leq \frac{\left|x_{0}-x\right|}{1-\lambda\left\langle x_{0}^{*}, x\right\rangle} \leq \frac{\sqrt{c\left(1-\left\langle x_{0}^{*}, x\right\rangle\right)}}{1-\lambda\left\langle x_{0}^{*}, x\right\rangle} \leq \frac{\sqrt{c}}{2 \sqrt{\lambda(1-\lambda)}} .
$$

The last inequality follows as for $t<1$ and $0<\lambda<1$

$$
\frac{\sqrt{1-t}}{1-\lambda t} \leq \frac{1}{2 \sqrt{\lambda(1-\lambda)}}
$$

Proposition 1. Let $K \in \mathcal{K}_{n}$ be in $C_{2}^{+}$and such that $0 \in \operatorname{int}(K)$. Let $p$ be a proper affine invariant point. Then there is a constant $C$ such that for all $\lambda$ with $\frac{1}{2} \leq \lambda<1$, for all $x_{0}^{*} \in \partial K^{\circ}$ and all $v \in S^{n-1}$ with $\left\langle x_{0}, v\right\rangle=0$

$$
\frac{\left\langle p_{\lambda}, \frac{x_{0}}{\left|x_{0}\right|}\right\rangle}{\left|\left\langle p_{\lambda}, v\right\rangle\right|} \geq \frac{C}{\sqrt{1-\lambda}}
$$

where $x_{0} \in \partial K$ is the unique point with $\left\langle x_{0}, x_{0}^{*}\right\rangle=1$. In particular, uniformly in $x_{0}^{*} \in$ $\partial K^{\circ}$,

$$
\lim _{\lambda \rightarrow 1} \frac{p\left(\left(K^{\circ}-\lambda x_{0}^{*}\right)^{\circ}\right)}{\left|p\left(\left(K^{\circ}-\lambda x_{0}^{*}\right)^{\circ}\right)\right|}=\frac{x_{0}}{\left|x_{0}\right|} .
$$


Proof. Let $u=\frac{x_{0}}{\left|x_{0}\right|} \in S^{n-1}, u^{*}=\frac{x_{0}^{*}}{\left|x_{0}^{*}\right|} \in S^{n-1}$ and for $0<\lambda<1$ let $p_{\lambda}=p\left(\left(K^{\circ}-\right.\right.$ $\left.\lambda x_{0}^{*}\right)^{\circ}$ ). To show (8), we choose $w \in S^{n-1}$ such that

$$
u^{*}=\left\langle u^{*}, u\right\rangle u+\sqrt{1-\left\langle u^{*}, u\right\rangle^{2}} w .
$$

Please note that $\langle w, u\rangle=0$. Then

$$
\left\langle p_{\lambda}, u\right\rangle=\frac{1}{\left\langle u^{*}, u\right\rangle}\left(\left\langle p_{\lambda}, u^{*}\right\rangle-\sqrt{1-\left\langle u^{*}, u\right\rangle^{2}}\left\langle p_{\lambda}, w\right\rangle\right) .
$$

By Lemmas 5 and 7 for $\lambda$ big enough, $p$ is proper and all $v \in S^{n-1}$ with $\left\langle x_{0}, v\right\rangle=0$

$$
\frac{\left\langle p_{\lambda}, u\right\rangle}{\left|\left\langle p_{\lambda}, v\right\rangle\right|} \geq \frac{\frac{1}{\left\langle u^{*}, u\right\rangle}\left(\frac{c}{1-\lambda}-\sqrt{1-\left\langle u^{*}, u\right\rangle^{2}} \frac{d}{2 \sqrt{\lambda(1-\lambda)}}\right)}{\frac{d}{2 \sqrt{\lambda(1-\lambda)}}}
$$

where $d$ denotes the constant from Lemma 7. Since

$$
\frac{1}{\left\langle u^{*}, u\right\rangle} \geq \min _{x_{0}^{*} \in \partial K^{\circ}}\left|x_{0}^{*}\right|\left|x_{0}\right|>0
$$

there is a constant $C$ such that for all $\lambda$ with $\frac{1}{2} \leq \lambda<1$ and all $x_{0} \in \partial K$ and $x_{0}^{*} \in \partial K^{\circ}$ with $\left\langle x_{0}, x_{0}^{*}\right\rangle=1$

$$
\frac{\left\langle p_{\lambda}, u\right\rangle}{\left|\left\langle p_{\lambda}, v\right\rangle\right|} \geq \frac{C}{\sqrt{1-\lambda}}
$$

By Lemma 5 , $\left|p_{\lambda}\right| \rightarrow+\infty$ when $\lambda \rightarrow 1$. We write $p_{\lambda}=\left\langle p_{\lambda}, u\right\rangle u+\left(p_{\lambda}-\left\langle p_{\lambda}, u\right\rangle u\right)$ and put $v=\frac{p_{\lambda}-\left\langle p_{\lambda}, u\right\rangle u}{\left|p_{\lambda}+\left\langle p_{\lambda}, u\right\rangle u\right|}$. Then $v$ is orthogonal to $u$ and

$$
\frac{p_{\lambda}}{\left|p_{\lambda}\right|}=\frac{\left\langle p_{\lambda}, u\right\rangle u+\left\langle p_{\lambda}, v\right\rangle v}{\left[\left(\left\langle p_{\lambda}, u\right\rangle\right)^{2}+\left(\left\langle p_{\lambda}, v\right\rangle\right)^{2}\right]^{\frac{1}{2}}}=\frac{u}{\left[1+\frac{\left(\left\langle p_{\lambda}, v\right\rangle\right)^{2}}{\left(\left\langle p_{\lambda}, u\right\rangle\right)^{2}}\right]^{\frac{1}{2}}}+\frac{v}{\left[1+\frac{\left(\left\langle p_{\lambda}, u\right\rangle\right)^{2}}{\left(\left\langle p_{\lambda}, v\right\rangle\right)^{2}}\right]^{\frac{1}{2}}},
$$

which converges to $u$ if (8) holds.

Proposition 2. Let $p \in \mathfrak{P}_{n}$ be proper. For $C \in \mathcal{K}_{n}$, let $F: \operatorname{int}(C) \rightarrow \mathbb{R}^{n}$ be the mapping defined by

$$
F(z)=p\left((C-z)^{\circ}\right) .
$$

Then $F$ is surjective.

Proof. We fix $z_{0} \in \operatorname{int}(C)$ and put $L=\left(C-z_{0}\right)^{\circ}$. Then $0 \in \operatorname{int}(L)$ and $L^{\circ}=C-z_{0}$, so that the statement of the proposition is equivalent to:

For any $K \in \mathcal{K}_{n}$ such that $0 \in \operatorname{int}(K)$, the mapping $F: \operatorname{int}\left(K^{\circ}\right) \rightarrow \mathbb{R}^{n}$ defined by $F\left(y^{*}\right)=p\left(\left(K^{\circ}-y^{*}\right)^{\circ}\right)$ is surjective.

We shall prove it in this form.

We first treat the case when the body $K$ is $C_{2}^{+}$. Suppose that $F$ is not surjective. Then for some $x \in \mathbb{R}^{n}$, one has $F\left(y^{*}\right) \neq x$ for every $y^{*} \in \operatorname{int}\left(K^{\circ}\right)$. We define the function $G: \operatorname{int}\left(K^{\circ}\right) \rightarrow S^{n-1}$ by

$$
G\left(y^{*}\right)=\frac{F\left(y^{*}\right)-x}{\left|F\left(y^{*}\right)-x\right|} .
$$


By Lemma $\left|F\left(y^{*}\right)\right| \rightarrow \infty$ for $y^{*} \rightarrow \partial K^{\circ}$. By this and by Proposition 1 we have for all $y^{*} \in \operatorname{int}\left(K^{\circ}\right)$,

$$
\lim _{y^{*} \rightarrow \partial K^{\circ}} \frac{F\left(y^{*}\right)-x}{\left|F\left(y^{*}\right)-x\right|}=\frac{y}{|y|}
$$

where $y$ is the unique point in $\partial K$ such that $\left\langle y^{*}, y\right\rangle=1$. Thus $G$ can be extended to a continuous function $H: K^{\circ} \rightarrow S^{n-1}$, setting

$$
H\left(y^{*}\right)=\left\{\begin{array}{cl}
G\left(y^{*}\right) & \text { if } y^{*} \in \operatorname{int}\left(K^{\circ}\right) \\
\frac{y}{|y|} & \text { if } y^{*} \in \partial K^{\circ}
\end{array}\right.
$$

Indeed, $H$ is continuous on $\partial K^{\circ}$ since $\partial K$ is $C_{+}^{2}$. We also define a continuous function $\theta: B_{2}^{n} \rightarrow K^{\circ}$ by

$$
\theta(z)=\left\{\begin{array}{cc}
0 & \text { if } z=0 \\
\frac{|z|}{h_{K}(z)} z & \text { if } z \in B_{2}^{n} \backslash\{0\} .
\end{array}\right.
$$

Then $\phi=H \circ \theta: B_{2}^{n} \rightarrow S^{n-1}$ is continuous. It follows that the function $\psi: B_{2}^{n} \rightarrow B_{2}^{n}$ defined by

$$
\psi(z)=\frac{z-\phi(z)}{2}
$$

is also continuous. By the Brouwer fixed point theorem (see e.g. [16]), for some $z_{0} \in B_{2}^{n}$, one has $\psi\left(z_{0}\right)=z_{0}$, so that

$$
z_{0}=-\phi\left(z_{0}\right) \in S^{n-1} .
$$

$z_{0} \neq 0$ since $z_{0} \in S^{n-1}$. Therefore

$$
\theta\left(z_{0}\right)=\frac{\left|z_{0}\right| z_{0}}{h_{K}\left(z_{0}\right)}
$$

and consequently $x_{0}^{*}=\theta\left(z_{0}\right)=\frac{\left|z_{0}\right| z_{0}}{h_{K}\left(z_{0}\right)} \in \partial K^{\circ}$. One gets

$$
\phi\left(z_{0}\right)=H\left(x_{0}^{*}\right)=\frac{x_{0}}{\left|x_{0}\right|},
$$

where $x_{0}$ is the unique point in $\partial K$ such that $\left\langle x_{0}^{*}, x_{0}\right\rangle=1$. Since

$$
z_{0}=\frac{x_{0}^{*}}{\left|x_{0}^{*}\right|}=-\phi\left(z_{0}\right)=-\frac{x_{0}}{\left|x_{0}\right|},
$$

we get

$$
\left\langle z_{0}, z_{0}\right\rangle=\left\langle z_{0},-\phi\left(z_{0}\right)\right\rangle=-\left\langle\frac{x_{0}}{\left|x_{0}\right|}, \frac{x_{0}^{*}}{\left|x_{0}^{*}\right|}\right\rangle=-\frac{1}{\left|x_{0}\right|\left|x_{0}^{*}\right|}<0,
$$

which is absurd.

Now we treat the general case. Let $K$ be a convex body such that $0 \in \operatorname{int}(K)$. Then, there exists a sequence $\left(K_{m}\right)$ of $C_{2}^{+}$convex bodies converging to $K$ in the Hausdorff metric. For $m$ big enough, one has $0 \in \operatorname{int}\left(K_{m}\right)$. Fix $x \in \mathbb{R}^{n}$. By above, there exists $y_{m}^{*} \in \operatorname{int}\left(K_{m}^{\circ}\right)$ such that $p\left(\left(K_{m}^{\circ}-y_{m}^{*}\right)^{\circ}\right)=x$. Since $K_{m} \rightarrow K, K_{m}^{\circ} \rightarrow K^{\circ}$. Let $y_{m_{k}}^{*} \rightarrow y^{*}$ be a converging subsequence of the sequence $\left(y_{m}^{*}\right)_{m \in \mathbb{N}}$. Then it is easy to see that $y^{*} \in \operatorname{int}\left(K^{\circ}\right)$, and, by continuity, $p\left(\left(K^{\circ}-y^{*}\right)^{\circ}\right)=x$. 


\section{The main theorems.}

The first theorem in this section follows immediately from Lemma 2 and Proposition 2 Observe however that the statement of Proposition 2 is stronger than surjectivity of all proper $p \in \mathfrak{P}_{n}$.

Theorem 1. Every proper affine invariant point is surjective.

While every proper affine invariant point is surjective, for injectivity this is not the case. This is the content of the next theorem.

Theorem 2. For $n \geq 2$, there exists $p \in \mathfrak{P}_{n}$ such that $p$ is not injective.

We postpone the proof of Theorem 2 to the end of this section. First we apply Theorem 1 to obtain the following result.

Theorem 3. Let $p \in \mathfrak{P}_{n}$ be proper. Then the following assertions are equivalent.

(i) $p$ has a dual $q$.

(ii) There exists a proper $r \in \mathfrak{P}_{n}$ such that $p$ is a dual of $r$.

(iii) $p$ is injective.

Moreover, if one of these assertions hold, then $p$ has a unique dual point $p^{\circ} \cdot p^{\circ}$ is proper, $r=p^{\circ}$ and $p^{\circ}$ has a unique dual which is $p$.

\subsection{Proof of Theorem 3 ,}

We shall need more lemmas.

Lemma 8. Let $K \in \mathcal{K}_{n}$ and suppose that $K-y \subset \beta(y-K)$ for some $\beta>1$ and some $y \in \mathbb{R}^{n}$. For all $x \in \mathbb{R}^{n}$ and for all real numbers $\alpha$ and $\gamma$ such that $0<\alpha<1<\gamma$ the following assertions hold.

(i) If $x-y \in \alpha(K-y)$, then $K-x \subset \frac{\beta+\alpha}{1-\alpha}(x-K)$.

(ii) If $K-x \subset \gamma(x-K)$, then $x-y \in \frac{\beta \gamma-1}{\beta(\gamma+1)}(K-y)$.

Proof. We may assume that $y=0$. Otherwise we consider the body $K^{\prime}=K-y$.

(i) Suppose $x \in \alpha K$. Let $\delta=\frac{\beta+\alpha}{1-\alpha}$. We need to prove that

$$
(\delta+1) x-K \subset \delta K .
$$

With the assumption in (i), the inclusion $-K \subset \beta K$ and the convexity of $K$, we get

$$
(\delta+1) x-K \subset \alpha(\delta+1) K+\beta K=\left(\alpha\left(1+\frac{\beta+\alpha}{1-\alpha}\right)+\beta\right) K=\delta K .
$$

(ii) Suppose that $K-x \subset \gamma(x-K)$. Then $(\gamma+1) x-K \subset \gamma K$. Now we use that $\frac{K}{\beta} \subset-K$, divide by $\gamma$ and get with $y=\frac{\gamma+1}{\gamma} x$ and $t=\frac{1}{\beta \gamma}$ that

$$
y+t K \subset K \text {. }
$$


Since $K$ is bounded and closed, it follows that $y \in(1-t) K$, so that

$$
x=\frac{\gamma}{\gamma+1} y \in \frac{\beta \gamma-1}{\beta(\gamma+1)} K .
$$

Lemma 9. Let $L \in \mathcal{K}_{n}$ and let $0<r \leq R<\infty$ be such that $r B_{2}^{n} \subset L \subset R B_{2}^{n}$. Suppose that there is $x \in \mathbb{R}^{n}$ and $\gamma \geq 1$ such that $L-x \subset \gamma(x-L)$. Then

$$
\frac{2 r}{\gamma+1} B_{2}^{n} \subset L-x \subset \frac{2 \gamma R}{\gamma+1} B_{2}^{n}
$$

Proof. As $L-x \subset \gamma(x-L)$,

$$
2 r B_{2}^{n} \subset L-L=L-x+x-L \subset \gamma(x-L)+x-L=(\gamma+1)(x-L) .
$$

This gives the first inclusion. The second one is obtained from

$$
(\gamma+1)(L-x)=\gamma(L-x)+L-x \subset \gamma(L-x)-\gamma(L-x)=\gamma(L-L) \subset 2 \gamma R B_{2}^{n} .
$$

For the next lemma, recall (5) where we introduced the notation $\phi_{p}(K)=K^{p(K)}$.

Lemma 10. Let $p$ be a proper affine invariant point and suppose that the mapping $\phi_{p}$ is bijective. Let $q: \mathcal{K}_{n} \rightarrow \mathbb{R}^{n}$ be defined by $q(L)=p(K)$ whenever $L=K^{p(K)}$. Then $\phi_{p}$ is a homeomorphism and the mapping $q$ is continuous.

Proof. It is clear that $\phi_{p}$ is continuous. We want to show that $\phi_{p}^{-1}$ is continuous. We use now that the inverse of a continuous, bijective map between locally compact Hausdorff spaces is continuous if the inverse image of any compact set is compact. Since $\mathcal{K}_{n}$ endowed with the Hausdorff metric is locally compact, it is enough to verify that for any compact subset $\mathcal{L}$ of $\mathcal{K}_{n},\left(\phi_{p}\right)^{-1}(\mathcal{L})$ is compact.

By Proposition 1 of [13, one has for every $K \in \mathcal{K}_{n}$ that

$$
p(K)-g(K) \subset \alpha((K-g(K)),
$$

for some $0<\alpha<1$. Here, $g$ denotes the centroid. It is well known that

$$
K-g(K) \subset n(g(K)-K) .
$$

It follows from Lemma 8 (i)

$$
K-p(K) \subset \frac{n+\alpha}{1-\alpha}(p(K)-K),
$$

whence

$$
(K-p(K))^{\circ} \subset-\frac{n+\alpha}{1-\alpha}(K-p(K))^{\circ} .
$$

Now $\phi_{p}(K)=\left((K-p(K))^{\circ}+p(K)\right.$, so that

$$
\phi_{p}(K)-p(K) \subset \frac{n+\alpha}{1-\alpha}\left(p(K)-\phi_{p}(K)\right) .
$$


Let $\mathcal{L}$ be a compact subset of $\mathcal{K}_{n}$. By affine invariance, we may suppose that there are $0<r \leq R<\infty$ such that for every $L \in \mathcal{L}$

$$
r B_{2}^{n} \subset L \subset R B_{2}^{n} .
$$

Let $L \in \mathcal{L}$ and $K=\left(\phi_{p}\right)^{-1}(L)$, that is,

$$
L-p(K)=\phi_{p}(K)-p(K) \subset \frac{n+\alpha}{1-\alpha}\left(p(K)-\phi_{p}(K)\right)=\frac{n+\alpha}{1-\alpha}(p(K)-L) .
$$

The last inclusion follows from (9). Therefore, by Lemma 9 with $\gamma=\frac{n+\alpha}{1-\alpha}$,

$$
\frac{2 r}{\gamma+1} B_{2}^{n} \subset L-p(K) \subset \frac{2 \gamma R}{\gamma+1} B_{2}^{n} .
$$

By duality

$$
\frac{\gamma+1}{2 \gamma R} B_{2}^{n} \subset K-p(K) \subset \frac{\gamma+1}{2 r} B_{2}^{n} .
$$

As $p$ is proper, $p(K) \in L \subset R B_{2}^{n}$. Hence we get that for some $0<c<d$, one has

$$
\left(\phi_{p}\right)^{-1}(\mathcal{L}) \subset \mathcal{L}^{\prime}=\left\{K \in \mathcal{K}_{n}: x+c B_{2}^{n} \subset K \subset d B_{2}^{n} \text { for some } x \in \mathbb{R}^{n}\right\} .
$$

Now, it is easily seen that $\mathcal{L}^{\prime}$ is a compact subset of $\mathcal{K}_{n}$, and consequently $\left(\phi_{p}\right)^{-1}(\mathcal{L})$ is as a closed subset of a compact set compact.

\section{Proof of Theorem 3,}

(i) $\Longrightarrow$ (iii) Suppose that $K_{1}, K_{2} \in \mathcal{K}_{n}$ satisfy $K_{1}^{p\left(K_{1}\right)}=K_{2}^{p\left(K_{2}\right)}$. Then, by the definition of dual point,

$$
p\left(K_{1}\right)=q\left(K_{1}^{p\left(K_{1}\right)}\right)=q\left(K_{1}^{p\left(K_{1}\right)}\right)=p\left(K_{2}\right) .
$$

(iii) $\Longrightarrow$ (i) By Theorem 1, $p$ is surjective and consequently bijective. By Remark 1(i), this is equivalent that $\phi_{p}$ is bijective. Lemma 10 then gives

$$
q(L)=q\left(K^{p(K)}\right)=p(K)
$$

and thus $q$ is the dual of $p$.

(i) $\Longrightarrow$ (ii) We first show that $q$ is proper. By Theorem $1, p$ is surjective. Hence there exists $K \in \mathcal{K}_{n}$ if $C \in \mathcal{K}_{n}$ such that $C=K^{p(K)}$. As $q$ is the dual of $p$, one has $q(C)=q\left(K^{p(K)}\right)=p(K)$ and thus $C=K^{p(K)}=K^{q(C)}$. By the bipolar theorem (1), $C^{q(C)}=\left(K^{q(C)}\right)^{q(C)}=K$, which proves that $q(C) \in \operatorname{int}(C)$. Next we show:

If a proper $p \in \mathcal{P}_{n}$ has a dual $q$, then $p$ is a dual of $q$.

Again, by Theorem 1, for all $L \in \mathcal{K}_{n}$, one has $L=K^{p(K)}$ for some $K \in \mathcal{K}_{n}$. Thus, using the definition of duality, $p(K)=q\left(K^{p(K)}\right)$ and the bipolar theorem (1),

$$
L^{q(L)}=\left(K^{p(K)}\right)^{q\left(K^{p(K)}\right)}=\left(K^{p(K)}\right)^{p(K)}=K
$$

and hence $p\left(L^{q(L)}\right)=p(K)=q(L)$, which proves that $p$ is a dual of $q$. Thus we can take $r=q$ in (ii).

(ii) $\Longrightarrow$ (i) This follows immediately with (10). 


\subsection{A product mapping on affine invariant points.}

The next definition will turn out to be useful to characterize duality. There, $I_{n}: \mathfrak{P}_{n} \rightarrow$ $\mathfrak{P}_{n}$ denotes the identity map. Recall also the mapping $\phi_{p}: \mathcal{K}_{n} \rightarrow \mathcal{K}_{n}$ defined in (5) by $\phi_{p}(K)=K^{p(K)}$.

Definition 5. Let $(p, q) \in \mathfrak{P}_{n} \times \mathfrak{P}_{n}$ be such $p$ and $q$ are proper. We define the map $[p, q]: \mathfrak{P}_{n} \rightarrow \mathfrak{P}_{n}$ by

$$
[p, q](r)=r \circ \phi_{q} \circ \phi_{p}-q \circ \phi_{p}+p .
$$

We show first that $[p, q](r)$ is indeed in $\mathfrak{P}_{n}$.

Since $\phi_{p}$ is continuous for all proper $p \in \mathfrak{P}_{n},[p, q](r)$ is continuous for all $r \in \mathfrak{P}_{n}$.

Now we address affine invariance. Let $b \in \mathbb{R}^{n}, T: \mathbb{R}^{n} \rightarrow \mathbb{R}^{n}$ be a bijective linear map and $S: \mathbb{R}^{n} \rightarrow \mathbb{R}^{n}$ be defined by $S x=T x+b$. Put $L=(K-p(K))^{\circ}$. By definition, for all $K \in \mathcal{K}_{n}$,

$$
[p, q](r)(K)=r\left((L-q(L))^{\circ}\right)+p(K) .
$$

We put $L^{\prime}=(S(K)-p(S(K)))^{\circ}$. Then with (2),

$$
L^{\prime}=(T(K)-p(T(K)))^{\circ}=(T(K-p(K)))^{\circ}=\left(T\left(L^{\circ}\right)\right)^{\circ}=T^{*-1}(L) .
$$

Thus, again with (2),

$$
\left(L^{\prime}-q\left(L^{\prime}\right)\right)^{\circ}=\left(T^{*-1}(L)-q\left(T^{*-1}(L)\right)\right)^{\circ}=\left(T^{*-1}(L-q(L))\right)^{\circ}=T\left((L-q(L))^{\circ}\right),
$$

so that

$$
\begin{aligned}
{[p, q](r)(S(K)) } & =r\left(\left(L^{\prime}-q\left(L^{\prime}\right)\right)^{\circ}\right)+p(S(K))=r\left(T\left((L-q(L))^{\circ}\right)\right)+p(T(K))+b \\
& =T\left(r\left((L-q(L))^{\circ}\right)+p(K)\right)+b=S([p, q](r)(K)) .
\end{aligned}
$$

With the aid of $[p, q]$, we give another characterization of dual affine invariant points.

Proposition 3. Let $p, q \in \mathfrak{P}_{n}$ be proper. The following are equivalent.

(i) $[p, q]=I_{n}$.

(ii) $[p, q](p)=p$.

(iii) $q$ is the dual of $p$.

(iv) $p$ is the dual of $q$.

Proof. It is clear that (i) $\Longrightarrow$ (ii) and by Theorem 3 (iii) is equivalent to (iv). We now show the remaining implications.

(ii) $\Longrightarrow$ (iv) By Theorem 1, $p$ is surjective. Hence for every $L \in \mathcal{K}_{n}$ there is $K \in \mathcal{K}_{n}$ such that $L=\phi_{p}(K)$. By (ii),

$$
p(K)=[p, q](p)(K)=\left(p \circ \phi_{q} \circ \phi_{p}\right)(K)-\left(q \circ \phi_{p}\right)(K)+p(K),
$$


which is equivalent to

$$
p\left(\left(\phi_{p}(K)\right)^{q\left(\phi_{p}(K)\right)}\right)-q\left(\phi_{p}(K)\right)=0,
$$

and again, equivalent to

$$
p\left(L^{q(L)}\right)=q(L) .
$$

By the definition, this means that $p$ is the dual of $q$.

(iii) $\Longrightarrow$ (i) If $q$ is the dual of $p$, then $q\left(K^{p(K)}\right)=p(K)$ for every $K \in \mathcal{K}_{n}$. This means that $q \circ \phi_{p}=p$. It follows that

$$
\left(\phi_{q} \circ \phi_{p}\right)(K)=\left(\phi_{p}(K)\right)^{q\left(\phi_{p}(K)\right)}=\left(\phi_{p}(K)\right)^{p(K)}=\left(K^{p(K)}\right)^{p(K)}=K .
$$

The last equality follows from the Bipolar Theorem. One has thus

$$
[p, q](r)=r \circ \phi_{q} \circ \phi_{p}-q \circ \phi_{p}+p=r-p+p=r .
$$

The next proposition describes the product $\left[p_{1}, q_{1}\right] \circ\left[p_{2}, q_{2}\right]$ in some special cases. Note also that

$$
\phi_{p} \circ \phi_{p^{\circ}}=\phi_{p^{\circ}} \circ \phi_{p}=I_{n}, \quad p=p^{\circ} \circ \phi_{p} \text { and } p^{\circ}=p \circ \phi_{p^{\circ}} .
$$

Proposition 4. Let $p, r, s \in \mathfrak{P}_{n}$ be proper and suppose that $p$ has a dual $p^{\circ}$. Then

$$
\left[r, p^{\circ}\right] \circ[p, s]=[r, s] .
$$

In particular, if $p, q \in \mathfrak{P}_{n}$ are proper and have dual points $p^{\circ}$ and $q^{\circ}$, then

$$
\left[q^{\circ}, p^{\circ}\right] \circ[p, q]=I_{n} .
$$

Proof. Let $t \in \mathfrak{P}_{n}$. Then with (11),

$$
\begin{aligned}
& \left(\left[r, p^{\circ}\right] \circ[p, s]\right)(t) \\
& =[p, s](t) \circ \phi_{p^{\circ}} \circ \phi_{r}-p^{\circ} \circ \phi_{r}+r \\
& =\left(t \circ \phi_{s} \circ \phi_{p}-s \circ \phi_{p}+p\right) \circ \phi_{p^{\circ}} \circ \phi_{r}-p^{\circ} \circ \phi_{r}+r \\
& =t \circ \phi_{s} \circ \phi_{p} \circ \phi_{p^{\circ}} \circ \phi_{r}-s \circ \phi_{p} \circ \phi_{p^{\circ}} \circ \phi_{r}+p \circ \phi_{p^{\circ}} \circ \phi_{r}-p^{\circ} \circ \phi_{r}+r \\
& =t \circ \phi_{s} \circ \phi_{r}-s \circ \phi_{r}+p^{\circ} \circ \phi_{r}-p^{\circ} \circ \phi_{r}+r \\
& =t \circ \phi_{s} \circ \phi_{r}-s \circ \phi_{r}+r=[r, s](t) \text {. }
\end{aligned}
$$

Therefore $\left[r, p^{\circ}\right] \circ[p, s]=[r, s]$ and $\left[q^{\circ}, p^{\circ}\right] \circ[p, q]=\left[q^{\circ}, q\right]=I_{n}$. The last equality follows by Proposition 3

Remark 3. It would be interesting to know more about $[p, p]$ and

$$
\left.[p, p]^{k}(p):=[p, p] \circ \cdots \circ[p, p]\right)(p), k \text { times, } k \geq 1 .
$$

Is there a limit for $k \rightarrow \infty$ ? 
Let $\mathfrak{A}_{n}$ be as in Definition 2 and let $A \in \mathfrak{A}_{n}$. Let $p, a$ and $b$ be in $\mathfrak{P}_{n}$. Suppose in addition that $p$ is proper and that $a(M) \in \operatorname{int}(A(M))$ for any $M \in \mathcal{K}_{n}$. For $K \in \mathcal{K}_{n}$, we define

$$
B(K)=\left(A\left((K-p(K))^{\circ}\right)-a\left((K-p(K))^{\circ}\right)\right)^{\circ}+b(K) .
$$

Then $B$ is an affine invariant set mapping, i.e. $B \in \mathfrak{A}_{n}$. We now show this.

It is clear from the hypothesis on $a$ that $B(K) \in \mathcal{K}_{n}$ and that $B$ is continuous. We prove next that $B$ is an affine invariant mapping. To do so, fix $K \in \mathcal{K}_{n}$ and put $M=(K-p(K))^{\circ}$. Then

$$
(B(K)-b(K))^{\circ}=A(M)-a(M),
$$

which shows that $b(K) \in \operatorname{int}(B(K))$. Let $T: \mathbb{R}^{n} \rightarrow \mathbb{R}^{n}$ be linear and one-to-one, $c \in \mathbb{R}^{n}$ and $S=T+c$. With (2),

$$
\begin{aligned}
(S(K)-p(S(K)))^{\circ} & =(T(K)+c-p(T(K)+c))^{\circ}=(T(K-p(K)))^{\circ} \\
& =T^{*-1}\left((K-p(K))^{\circ}\right)=T^{*-1}(M) .
\end{aligned}
$$

We get from this and (13) with $S(K)$ instead of $K$,

$$
(B(S(K))-b(S(K)))^{\circ}=A\left(T^{*-1}(M)\right)-a\left(T^{*-1}(M)\right)=T^{*-1}(A(M)-a(M)) .
$$

It follows with (2) and (13) that

$$
\begin{aligned}
B(S(K))-b(S(K)) & =T\left((A(M)-a(M))^{\circ}\right)=T(B(K)-b(K)) \\
& =T(B(K))+c-T(b(K))-c=S(B(K))-b(S(K)),
\end{aligned}
$$

so that $B(S(K))=S(B(K))$.

Suppose now that $p$ has a dual $p^{\circ}$. By Theorem $3 p^{\circ}$ is surjective. Hence for all $K \in \mathcal{K}_{n}$ there is $L \in \mathcal{K}_{n}$ such that $K=L^{p^{\circ}(L)}$. Using also (11), this implies that

$$
p(K)=p\left(L^{p^{\circ}(L)}\right)=\left(p \circ \phi_{p^{\circ}}\right)(L)=p^{\circ}(L),
$$

and

$$
(K-p(K))^{\circ}=\phi_{p}(K)-p(K)=\left(\phi_{p} \circ \phi_{p^{\circ}}\right)(L)=L-p^{\circ}(L) .
$$

One has then, also with (12),

$$
\begin{aligned}
B(K)-b(K) & =\left(A\left((K-p(K))^{\circ}\right)-a\left((K-p(K))^{\circ}\right)\right)^{\circ} \\
& =\left((A(L)-a(L))^{\circ} .\right.
\end{aligned}
$$

It follows that

$$
A(L)=(B(K)-b(K))^{\circ}+a(L)=\left(B\left(\left(L-p^{\circ}(L)\right)^{\circ}\right)-b\left(\left(L-p^{\circ}(L)\right)^{\circ}\right)\right)^{\circ}+a(L) .
$$

Thus we have proved the following proposition. 
Proposition 5. Let $A \in \mathfrak{A}_{n}$ and let $p, a$ and $b$ be in $\mathfrak{P}_{n}$. Suppose in addition that $p$ is proper and that $a(M) \in \operatorname{int}(A(M))$ for any $M \in \mathcal{K}_{n}$.

For $K \in \mathcal{K}_{n}, B$ defined by

$$
B(K)=\left(A\left((K-p(K))^{\circ}\right)-a\left((K-p(K))^{\circ}\right)\right)^{\circ}+b(K)
$$

is an affine invariant set mapping.

If $p$ has a dual point $p^{\circ}$, then $b(M) \in \operatorname{int}(B(M))$ for any $M \in \mathcal{K}_{n}$ and for any $L \in \mathcal{K}_{n}$,

$$
A(L)=\left(B\left(\left(L-p^{\circ}(L)\right)^{\circ}\right)-b\left(\left(L-p^{\circ}(L)\right)^{\circ}\right)\right)^{\circ}+a(L) .
$$

\subsection{Proof of Theorem 2 ,}

The proof is a consequence of the lemmas in this subsection. The last one gives the result.

Lemma 11. ([13], Lemma 6) Let $p \in \mathfrak{P}_{n}$ and let $g$ be the centroid. For $0<\varepsilon<1$, define $A_{p, \varepsilon}, B_{p, \varepsilon}: \mathcal{K}_{n} \rightarrow \mathcal{K}_{n}$ by

$$
A_{p, \varepsilon}(K)=\left\{x \in K \mid\left\langle x, p\left((K-g(K))^{\circ}\right)\right\rangle \geq \sup _{y \in K}\left\langle y, p\left((K-g(K))^{\circ}\right)\right\rangle-\varepsilon\right\} .
$$

and

$$
B_{p, \varepsilon}(K)=\left\{x \in K \mid\left\langle x, p\left((K-g(K))^{\circ}\right)\right\rangle \leq \inf _{y \in K}\left\langle y, p\left((K-g(K))^{\circ}\right)\right\rangle+\varepsilon\right\} .
$$

Then $A_{p, \varepsilon}$ and $B_{p, \varepsilon}$ are affine invariant set maps.

Remark 4. Since 0 is the Santaló point of $(K-g(K))^{\circ}, 0 \in \mathfrak{P}_{n}\left((K-g(K))^{\circ}\right)$. Therefore $\mathfrak{P}_{n}\left((K-g(K))^{\circ}\right)$ is a subspace of $\mathbb{R}^{n}$.

Lemma 12. Define $p_{\varepsilon, \delta}: \mathcal{K}_{n} \rightarrow \mathbb{R}^{n}$ by

$$
p_{\varepsilon, \delta}(K)=g\left(A_{g, \varepsilon}(K) \cup B_{g, \delta}(K)\right) .
$$

Then $p_{\varepsilon, \delta}$ is a proper affine invariant point.

Proof. The sets $A_{g, \varepsilon}(K)$ and $B_{g, \delta}(K)$ have non-empty interior. Therefore, $p_{\varepsilon, \delta}(K)$ is well defined and it is an interior point of $K$. By Lemma 11, for every bijective, affine mapping $T: \mathbb{R}^{n} \rightarrow \mathbb{R}^{n}$

$$
A_{g, \varepsilon}(T(K))=T\left(A_{p, \varepsilon}(K)\right) \quad \text { and } \quad B_{g, \delta}(T(K))=T\left(B_{g, \delta}(K)\right) .
$$

Therefore,

$$
A_{g, \varepsilon}(T(K)) \cup B_{g, \delta}(T(K))=T\left(A_{p, \varepsilon}(K) \cup B_{g, \delta}(K)\right) .
$$

It follows also from Lemma 11 that $p_{\varepsilon, \delta}$ is continuous. 
Remark 5. If $A_{g, \varepsilon}(K) \cap B_{g, \delta}(K)=\emptyset$, then

$$
\begin{aligned}
& g\left(A_{g, \varepsilon}(K) \cup B_{g, \delta}(K)\right) \\
& =\frac{\left|A_{g, \varepsilon}(K)\right|}{\left|A_{g, \varepsilon}(K)\right|+\left|B_{g, \delta}(K)\right|} g\left(A_{g, \varepsilon}(K)\right)+\frac{\mid B_{g, \delta}(K \mid}{\left|A_{p, \varepsilon}(K)\right|+\left|B_{g, \delta}(K)\right|} g\left(B_{g, \delta}(K)\right) .
\end{aligned}
$$

Lemma 13. For numbers $0<a<b$ let $K(a, b)$ be the convex body in $\mathbb{R}^{2}$ defined by

$$
K(a, b)=\operatorname{conv}\left\{\left(-\frac{\frac{2}{3} b+\frac{1}{3} a}{a+b}, \pm a\right),\left(\frac{\frac{2}{3} a+\frac{1}{3} b}{a+b}, \pm b\right)\right\} .
$$

Then

$$
g(K(a, b))=(0,0) \quad \text { and } \quad g\left(K(a, b)^{\circ}\right)=\left(\frac{-3 a b\left(b^{2}-a^{2}\right)}{\left(2 a^{2}+2 b^{2}+5 a b\right)\left(2 a^{2}+2 b^{2}+2 a b\right)}, 0\right) .
$$

In particular, the first coordinate of $g\left(K(a, b)^{\circ}\right)$ is negative.

Remark 6. Note that $K(a, b)$ is the translate of $\operatorname{conv}\{(0, \pm a),(1, \pm b)\}$ by $\left(-\frac{\frac{2}{3} b+\frac{1}{3} a}{a+b}, 0\right)$.

Proof. By symmetry the second coordinates of $g(K(a, b))$ and $g\left(K(a, b)^{\circ}\right)$ are 0 . That $g(K(a, b))=(0,0)$ follows from a simple computation. We see that

$K(a, b)=\left\{(x, y) \in \mathbb{R}^{2}:-\frac{\frac{2}{3} b+\frac{1}{3} a}{a+b} \leq x \leq \frac{\frac{2}{3} a+\frac{1}{3} b}{a+b},|y| \leq(b-a) x+\frac{2\left(a^{2}+b^{2}+a b\right)}{3(a+b)}\right\}$.

Hence

$K(a, b)^{\circ}=\operatorname{conv}\left\{\left(-\frac{3(a+b)}{a+2 b}, 0\right),\left(\frac{3(a+b)}{2 a+b}, 0\right),\left(\frac{-3\left(b^{2}-a^{2}\right)}{2 a^{2}+2 b^{2}+2 a b}, \frac{ \pm 3(b+a)}{2 a^{2}+2 b^{2}+2 a b}\right)\right\}$.

Therefore,

$$
\begin{aligned}
g\left(K(a, b)^{\circ}\right) & =\left(\frac{1}{3}\left(-\frac{a+b}{\frac{2}{3} b+\frac{1}{3} a}+\frac{a+b}{\frac{2}{3} a+\frac{1}{3} b}-3 \frac{b^{2}-a^{2}}{2 a^{2}+2 b^{2}+2 a b}\right), 0\right) \\
& =\left(\frac{-3 a b\left(b^{2}-a^{2}\right)}{\left(2 a^{2}+2 b^{2}+5 a b\right)\left(2 a^{2}+2 b^{2}+2 a b\right)}, 0\right) .
\end{aligned}
$$

In particular, the first coordinate of $g\left(K(a, b)^{\circ}\right)$ is negative.

Lemma 14. (i) For all $\varepsilon, \delta>0$, we have that $p_{\varepsilon, \delta}\left(B_{\infty}^{2}\right)=0$.

(ii) For all $\eta \in(0,1)$ there exist $\varepsilon>0$ and $\delta>0$ such that

$$
p_{\varepsilon, \delta}\left(\left(B_{\infty}^{2}\right)_{\eta e_{1}}\right)=0 .
$$

Here, $e_{1}=(1,0)$. 
Proof. (i) Since $B_{\infty}^{2}$ is 0 -symmetric, we have that $p_{\varepsilon, \delta}\left(B_{\infty}^{2}\right)=0$.

(ii) For $\eta$ with $0 \leq \eta<1$, let $B_{\eta}:=\left(B_{\infty}^{2}\right)_{\eta e_{1}}$. Then

$$
B_{\eta}=\operatorname{conv}\left\{\left(-\frac{1}{1+\eta}, \pm \frac{1}{1+\eta}\right),\left(\frac{1}{1-\eta}, \pm \frac{1}{1-\eta}\right)\right\} .
$$

We put $a=\frac{1}{1+\eta}$ and $b=\frac{1}{1-\eta}$ and get that $g\left(B_{\eta}\right)=\left(\frac{2(b-a)}{3}, 0\right)$. Hence

$$
B_{\eta}-g\left(B_{\eta}\right)=\operatorname{conv}\left\{\left(-\left(\frac{2}{3} b+\frac{1}{3} a\right), \pm a\right),\left(\frac{2}{3} a+\frac{1}{3} b \pm b\right)\right\} .
$$

Thus, with the notation of Lemma 13

$$
B_{\eta}-g\left(B_{\eta}\right)=T_{a, b}(K(a, b)),
$$

where $T_{a, b}: \mathbb{R}^{2} \rightarrow \mathbb{R}^{2}$ is defined by

$$
T_{a, b}(x, y)=((a+b) x, y) .
$$

It follows with (2) that

$$
\left(B_{\eta}-g\left(B_{\eta}\right)\right)^{\circ}=\left(T_{a, b}^{*}\right)^{-1}\left(K(a, b)^{\circ}\right) .
$$

Again by Lemma 13.

$$
g\left(\left(B_{\eta}-g\left(B_{\eta}\right)\right)^{\circ}\right)=\left(\frac{-3 a b(b-a)}{\left(2 a^{2}+2 b^{2}+5 a b\right)\left(2 a^{2}+2 b^{2}+2 a b\right)}, 0\right) .
$$

We replace $a=\frac{1}{1+\eta}$ and $b=\frac{1}{1-\eta}$ and set $G(\eta):=g\left(\left(B_{\eta}-g\left(B_{\eta}\right)\right)^{\circ}\right)$. Then

$$
G(\eta)=(\alpha(\eta), 0)=\left(\frac{-3 \eta\left(1-\eta^{2}\right)^{2}}{\left(3+\eta^{2}\right)\left(9-\eta^{2}\right)}, 0\right),
$$

with $\alpha(\eta)<0$ for every $\eta \in(0,1)$. Now we compute

$$
p_{\varepsilon, \delta}\left(B_{\eta}\right)=g\left(A_{g, \varepsilon}\left(B_{\eta}\right) \cup B_{g, \delta}\left(B_{\eta}\right)\right) .
$$

Since $\alpha(\eta)<0$ for $0<\eta<1$

$$
\begin{aligned}
A_{g, \varepsilon}\left(B_{\eta}\right) & =\left\{x \in B_{\eta} \mid\langle x, G(\eta)\rangle \geq \sup _{z \in B_{\eta}}\langle z, G(\eta)\rangle-\varepsilon\right\} \\
& =\left\{x \in B_{\eta} \mid\left\langle x, \alpha(\eta) e_{1}\right\rangle \geq \sup _{z \in B_{\eta}}\left\langle z, \alpha(\eta) e_{1}\right\rangle-\varepsilon\right\} \\
& =\left\{x \in B_{\eta}\left|\left\langle x,-e_{1}\right\rangle \geq \sup _{z \in B_{\eta}}\left\langle z,-e_{1}\right)\right\rangle-\frac{\varepsilon}{|\alpha(\eta)|}\right\} \\
& =\left\{\left(x_{1}, x_{2}\right) \in B_{\eta} \mid-x_{1} \geq \frac{1}{1+\eta}-\frac{\varepsilon}{|\alpha(\eta)|}\right\} \\
& =\left\{\left(x_{1}, x_{2}\right) \in B_{\eta} \mid x_{1} \leq-\frac{1}{1+\eta}+\frac{\varepsilon}{|\alpha(\eta)|}\right\} .
\end{aligned}
$$

Similarly,

$$
B_{g, \delta}\left(B_{\eta}\right)=\left\{x \in B_{\eta} \mid x_{1} \geq \frac{1}{1-\eta}-\frac{\delta}{|\alpha(\eta)|}\right\} .
$$


Please note that

$$
A_{g, \varepsilon}\left(B_{\eta}\right) \cap B_{g, \delta}\left(B_{\eta}\right)=\emptyset \text { if and only if } \varepsilon+\delta<\frac{2|\alpha(\eta)|}{1-\eta^{2}} .
$$

Suppose that we take $\varepsilon$ and $\delta$ so that this condition holds. By (14),

$$
p_{\varepsilon, \delta}\left(B_{\eta}\right)=\frac{\left|A_{g, \varepsilon}\left(B_{\eta}\right)\right| g\left(A_{g, \varepsilon}\left(B_{\eta}\right)\right)+\left|B_{g, \delta}\left(B_{\eta}\right)\right| g\left(B_{g, \delta}\left(B_{\eta}\right)\right)}{\left|A_{g, \varepsilon}\left(B_{\eta}\right)\right|+\left|B_{g, \delta}\left(B_{\eta}\right)\right|} .
$$

Thus $p_{\varepsilon, \delta}\left(B_{\eta}\right)=0$ if and only if

$$
0=\left|A_{g, \varepsilon}\left(B_{\eta}\right)\right| g\left(A_{g, \varepsilon}\left(B_{\eta}\right)\right)+\left|B_{g, \delta}\left(B_{\eta}\right)\right| g\left(B_{g, \delta}\left(B_{\eta}\right)\right)
$$

Since

$$
B_{\eta}=\left\{(x, y) \in \mathbb{R}^{2}\left|-\frac{1}{1+\eta} \leq x \leq \frac{1}{1-\eta},\right| y \mid \leq 1+\eta x\right\}
$$

and

$$
\left|A_{g, \varepsilon}\left(B_{\eta}\right)\right|=\frac{\varepsilon}{|\alpha(\eta)|}\left(\frac{2}{1+\eta}+\frac{\varepsilon \eta}{|\alpha(\eta)|}\right), \quad\left|B_{g, \delta}\left(B_{\eta}\right)\right|=\frac{\delta}{|\alpha(\eta)|}\left(\frac{2}{1-\eta}-\frac{\delta \eta}{|\alpha(\eta)|}\right),
$$

$p_{\varepsilon, \delta}\left(B_{\eta}\right)=0$ if and only if

$$
\begin{aligned}
0 & =\left(\frac{\varepsilon}{|\alpha(\eta)|}\left(\frac{2}{1+\eta}+\frac{\varepsilon \eta}{|\alpha(\eta)|}\right)\right) \int_{\left\{(x, y) \in B_{\eta}:-\frac{1}{1+\eta} \leq x \leq-\frac{1}{1+\eta}+\frac{\varepsilon}{\alpha \alpha(\eta)}\right\}} x d x d y \\
& +\left(\frac{\delta}{|\alpha(\eta)|}\left(\frac{2}{1-\eta}-\frac{\delta \eta}{|\alpha(\eta)|}\right)\right) \int_{\left\{(x, y) \in B_{\eta}: \frac{1}{1-\eta}-\frac{\delta}{\mid \alpha(\eta)} \leq x \leq \frac{1}{1-\eta}\right\}} x d x d y,
\end{aligned}
$$

which is equivalent to

$$
\begin{aligned}
0 & =\varepsilon^{2}\left(\frac{2}{1+\eta}+\frac{\varepsilon \eta}{|\alpha(\eta)|}\right)\left(-\frac{1}{(1+\eta)^{2}}+\frac{\varepsilon}{2|\alpha(\eta)|}\left(\frac{1-\eta}{1+\eta}+\frac{2 \varepsilon \eta}{3|\alpha(\eta)|}\right)\right) \\
& +\delta^{2}\left(\frac{2}{1-\eta}-\frac{\delta \eta}{|\alpha(\eta)|}\right)\left(\frac{1}{(1-\eta)^{2}}-\frac{\delta}{2|\alpha(\eta)|}\left(\frac{1+\eta}{1-\eta}-\frac{2 \delta \eta}{3|\alpha(\eta)|}\right)\right) .
\end{aligned}
$$

For $\varepsilon \leq \varepsilon_{0}<\frac{|\alpha(\eta)|}{1-\eta^{2}}$ fixed, put

$$
\begin{aligned}
f_{\varepsilon}(\delta)= & \varepsilon^{2}\left(\frac{2}{1+\eta}+\frac{\varepsilon \eta}{|\alpha(\eta)|}\right)\left(-\frac{1}{(1+\eta)^{2}}+\frac{\varepsilon}{2|\alpha(\eta)|}\left(\frac{1-\eta}{1+\eta}+\frac{2 \varepsilon \eta}{3|\alpha(\eta)|}\right)\right) \\
& +\delta^{2}\left(\frac{2}{1-\eta}-\frac{\delta \eta}{|\alpha(\eta)|}\right)\left(\frac{1}{(1-\eta)^{2}}-\frac{\delta}{2|\alpha(\eta)|}\left(\frac{1+\eta}{1-\eta}-\frac{2 \delta \eta}{3|\alpha(\eta)|}\right)\right) .
\end{aligned}
$$

Then, for sufficiently small $\epsilon$

$$
f_{\varepsilon}(\varepsilon)>0 \quad \text { and } \quad f_{\varepsilon}\left(\varepsilon^{2}\right)<0
$$

Thus, for all $\varepsilon \leq \varepsilon_{0}$, by the Intermediate Value Theorem, there exists $\varepsilon^{2}<\delta<\varepsilon$ with $f_{\varepsilon}(\delta)=0$, and hence $p_{\varepsilon, \delta}\left(B_{\eta}\right)=0$.

Proof of Theorem 2, Lemma 14 provides an example in dimension 2. This example is easily generalized to dimension $n$, with $B_{\infty}^{n}$ instead of $B_{\infty}^{2}$. 


\section{Examples of affine invariant points and sets.}

In this Section, we list some of the classical affine invariant points and sets, with proof if necessary. We will also introduce several new affine invariant points and sets. First, we state a lemma (the proof of which we leave to the reader) that provides a general tool to study those affine points which are given as minima or maxima of functions.

There, $\mathcal{C}_{0}(X)$ denotes the space of continuous functions on a locally compact metric space $X$, vanishing at $\infty$, endowed with the uniform norm $\|\cdot\|_{\infty}$.

Lemma 15. Let $\left(f_{n}\right)_{n \in \mathbb{N}} \subset \mathcal{C}_{0}(X)$ be a sequence of positive functions. Moreover assume that $\left\|f_{n}-f\right\|_{\infty} \rightarrow 0$, where $f \in \mathcal{C}_{0}(X)$ reaches its maximum at a unique point $x \in X$. Then for any sequence $\left(x_{n}\right)_{n \in \mathbb{N}}$ in $X$ such that $f_{n}$ reaches its maximum at $x_{n}$ for all $n$, one has $x_{n} \rightarrow x$ in $X$.

\section{The John regions of a convex body.}

Let $K \in \mathcal{K}_{n}$. Let $\mathcal{E}_{0}$ be the set of all ellipsoids in $\mathbb{R}^{n}$ centered at 0 . We define a function $f_{K}: \operatorname{int}(K) \rightarrow \mathbb{R}_{+}$by

$$
f_{K}(x)=\sup \left\{|E|: E \in \mathcal{E}_{0}, x+E \subset K\right\} .
$$

It is easy to see that this supremum is a maximum, that $f_{K}$ is continuous on $\operatorname{int}(K)$ and that $f_{K}(x) \rightarrow 0$ when $x \rightarrow \partial K$. Thus $f_{K}$ can be extended to the whole $\mathbb{R}^{n}$ as a continuous function with compact support, setting

$$
f_{K}(x)=0 \text { for } x \in \mathbb{R}^{n} \backslash \operatorname{int}(K) .
$$

We omit the proof of the following easy lemma.

Lemma 16. The mapping $K \rightarrow f_{K}$ is continuous from $\mathcal{K}_{n}$ to $\mathcal{C}_{0}\left(\mathbb{R}^{n}\right)$.

Let us recall the celebrated theorem by F. John (see e.g. [23).

Theorem 4. (F. John) Let $K \in \mathcal{K}_{n}$ and suppose that $B_{2}^{n} \subset K\left(\right.$ resp. $\left.K \subset B_{2}^{n}\right)$. The following are equivalent.

(i) $B_{2}^{n}$ is the ellipsoid of maximal volume contained in $K$ (resp. of minimal volume containing $K$ ).

(ii) There exist $u_{i} \in S^{n-1} \cap \partial K \cap \partial K^{*}$ and $c_{i}>0,1 \leq i \leq m \leq n(n+1)$, such that

$$
\sum_{i=1}^{m} c_{i} u_{i}=0 \quad \text { and } \quad x=\sum_{i=1}^{m} c_{i}\left\langle x, u_{i}\right\rangle u_{i} \quad \text { for every } x \in \mathbb{R}^{n} .
$$

Thus, there is a unique ellipsoid of maximal volume $J(K)$ contained in $K$, called the John ellipsoid of $K$, and a unique ellipsoid of minimal volume $L(K)$ containing $K$, called the Löwner ellipsoid of $K$. We call its centers respectively, $j(K)$, the John point of $K$ 
and $l(K)$, the Löwner point of $K$. With the previous notation, $j(K)$ is the unique point $x \in \operatorname{int}(K)$ such that $\left\|f_{K}\right\|_{\infty}=f_{K}(x)$.

Then the next proposition follows immediately from Lemmas 15 and 16 .

Proposition 6. $K \rightarrow j(K)$ is an affine invariant point.

The following lemma allows to say more.

Lemma 17. With the preceding notations, $f_{K}^{\frac{1}{n}}$ is concave on $\operatorname{int}(K)$ and hence $f_{K}$ is log-concave on $\mathbb{R}^{n}$.

Proof. For $i=1,2$, let $x_{i} \in \operatorname{int}(K)$ and $E_{i} \in \mathcal{E}_{0}$ such that $x_{i}+E_{i} \subset K$. After an affine transform, we may suppose that $E_{1}=B_{2}^{n}$ and $E_{2}=\Lambda\left(B_{2}^{n}\right)$, where $\Lambda: \mathbb{R}^{n} \rightarrow \mathbb{R}^{n}$ is a diagonal matrix with positive entries $\lambda_{1}, \ldots, \lambda_{n}$ on the diagonal.

For $t \in[0,1]$, define $E_{t}=((1-t) I d+t \Lambda)\left(B_{2}^{n}\right)$, where $I d$ is the identity matrix on $\mathbb{R}^{n}$. Then $E_{t} \in \mathcal{E}_{0}$ and

$$
(1-t) x_{1}+t x_{2}+E_{t} \subset(1-t)\left(x_{1}+E_{1}\right)+t\left(x_{2}+E_{2}\right) \subset K .
$$

By the Brunn-Minkowski inequality (e.g., [1], [18), we get

$$
f_{K}^{\frac{1}{n}}\left(t x_{1}+(1-t) x_{2}\right) \geq\left|E_{t}\right|^{\frac{1}{n}}=\left|(1-t) E_{1}+t E_{2}\right|^{\frac{1}{n}} \geq(1-t)\left|E_{1}\right|^{\frac{1}{n}}+t\left|E_{2}\right|^{\frac{1}{n}} .
$$

Definition 6. Let $c \in[0,1)$. We define the John region of $K$ of index $c$ by

$$
J_{c}(K)=\left\{x \in \mathbb{R}^{n}: f_{K}(x) \geq c\left\|f_{K}\right\|_{\infty}\right\} .
$$

By Lemma 17 $J_{c}(K)$ is convex. With Lemma 16] we then get the next proposition, which provides a new affine invariant set mapping.

Proposition 7. For $c \in(0,1)$, the mapping $K \rightarrow J_{c}(K)$ is a proper affine invariant set mapping from $\mathcal{K}_{n}$ to $\mathcal{K}_{n}$.

\section{The Löwner regions of a convex body.}

Let $K \in \mathcal{K}_{n}$. Define $\lambda_{K}: \mathbb{R}^{n} \rightarrow \mathbb{R}$ by

$$
\lambda_{K}(x)=\left(\inf \left\{\operatorname{vol}_{n}(E): E \in \mathcal{E}_{0} ; K \subset x+E\right\}\right)^{-1} .
$$

It is clear that $\lambda_{K}>0$ and $\lambda_{K} \in \mathcal{C}_{0}\left(\mathbb{R}^{n}\right)$. The following lemma is easy.

Lemma 18. The mapping $K \rightarrow \lambda_{K}$ is continuous from $\mathcal{K}_{n}$ to $\mathcal{C}_{0}\left(\mathbb{R}^{n}\right)$.

Since the center $l(K)$ of the Löwner ellipsoid $L(K)$ of $K$ is the unique point $x \in \operatorname{int}(K)$ such that $\left\|\lambda_{K}\right\|_{\infty}=\lambda_{K}(x)$, Lemmas [15 and 18 give the next proposition. 
Proposition 8. $K \rightarrow l(K)$ is an affine invariant point.

Definition 7. If $c \in[0,1)$, we define the Löwner region of $K$ of index $c$ by

$$
L_{c}(K)=\operatorname{conv}\left[\left\{x \in \mathbb{R}^{n}: \lambda_{K}(x) \geq c\left\|\lambda_{K}\right\|_{\infty}\right\}\right] .
$$

Proposition 9. $K \rightarrow L_{c}(K)$ is an affine invariant set mapping from $\mathcal{K}_{n}$ to $\mathcal{K}_{n}$.

In conclusion, Theorem 5 summarizes all these facts.

Theorem 5. Let $K \in \mathcal{K}_{n}$. If $j(K)$ and $l(K)$ denote respectively the centers of the John and of the Löwner ellipsoids of $K$, then $l$ and $j$ are in $\mathfrak{P}_{n}$ and $l=j^{\circ}$.

Proof. We only need to prove that $l\left(K^{j(K)}\right)=j(K)$, or, equivalently, that if the John ellipsoid $E_{K}$ of $K$ is centered at 0 , then the Löwner ellipsoid of $K^{\circ}$ is $\left(E_{K}\right)^{\circ}$. This follows from John's theorem.

Remark 7. Note that we need the full strength of John's theorem only to prove that $l=$ $j^{\circ}$. The fact that $j$ and $l$ are uniquely defined follows from more elementary reasonnings.

\section{The Santaló point and the center of gravity.}

The following result is well known (see e.g., [17]). There, $S_{K}: \operatorname{int}(K) \rightarrow \mathbb{R}^{n}$ is the function defined by $S_{K}(x)=\left|K^{x}\right|$.

Theorem 6. 117] Let $K \in \mathcal{K}_{n}$. Then the function $S_{K}$ is strictly log convex. Moreover $S_{K}(x) \rightarrow+\infty$ when $x \rightarrow \partial K$ and $S_{K}$ reaches its minimum at a unique point $s(K) \in$ $\operatorname{int}(K)$. This point is characterized by the fact that $s(K)$ is the centroid of $K^{s(K)}$ (or that 0 is the centroid of $\left.(K-s(K))^{\circ}\right)$.

Proposition 10. The mapping $K \rightarrow s(K)$ is a proper affine invariant point and $g=s^{\circ}$.

Proof. The uniqueness of $s(K)$ shows that $g$ is injective. Hence, by Theorem $3 g$ has a dual point $g^{\circ} \in \mathfrak{P}_{n}$. Thus $g^{\circ}\left(K^{g(K)}\right)=g(K)$, and by the preceding characterization, $s\left(K^{g(K)}\right)=g(K)$. Since $g$ is surjective by Theorem 1, it follows that $g^{\circ}=s \in \mathfrak{P}_{n}$.

Remark 8. (i) The fact that the mapping $\psi_{K}: \operatorname{int}(K) \rightarrow \mathbb{R}^{n}, \psi_{K}(x)=g\left(K^{x}\right)$ for $x \in$ $\operatorname{int}(K)$, is bijective can also be proved in an other way: The function $\Theta_{K}: \operatorname{int}(K) \rightarrow$ $(0,+\infty), \Theta_{K}(x)=\log S_{K}(x)$, is strictly convex and $\Theta(x) \rightarrow+\infty$ when $x \rightarrow \partial K$. It follows that $\nabla \Theta_{K}: \operatorname{int}(K) \rightarrow \mathbb{R}^{n}$ is bijective. Moreover, it is easily checked that

$$
\left(\nabla \Theta_{K}\right)(x)=g\left((K-x)^{\circ}\right) \text { for all } x \in \operatorname{int}(K) \text {. }
$$

(ii) The Santaló regions of $K$, defined in [11] , for $c>0$ by

$$
S_{c}(K)=\left\{z \in \operatorname{int}(K):\left|K^{z}\right| \leq(1+c)\left|K^{s(K)}\right|\right\}
$$

are affine invariant set mappings. 
The center of the maximal volume centrally symmetric body inside $K$.

The first part of the following proposition follows from the Brunn-Minkowski inequality, together with its equality case, the second part from Lemma 15.

Proposition 11. Let $K \in \mathcal{K}_{n}$. Then the function $\theta_{K}(x)=\operatorname{vol}_{n}(K \cap(2 x-K))^{\frac{1}{n}}$ is concave on its support and reaches its maximum at a unique point $m(K)$. Moreover, the mapping $m$ is a proper affine invariant point.

Proposition 12. Let $0<c<1$. For $K \in \mathcal{K}_{n}$, define

$$
M_{c}(K)=\left\{x \in \mathbb{R}^{n}:|K \cap(2 x-K)| \geq c|K \cap(2 m(K)-K)|\right\} .
$$

Then $K \rightarrow M_{c}(K)$ is an affine invariant set mapping.

Proof. As $\theta_{K}^{n}$ is concave on its support, $M_{c}(K)$ is convex. Affine invariance and continuity of the map $K \rightarrow M_{c}(K)$ are easy.

\section{The center of the maximal volume zonoid body inside $K$.}

Let $\mathcal{Z}$ be the (closed) set of zonoids in $\mathcal{K}_{n}$, and $\mathcal{Z}_{0}$ be the set of all zonoids that are centered at 0 (see[1] or [18). For $K \in \mathcal{K}_{n}$, let

$$
g_{K}(x)=\left\{\begin{array}{cc}
\max \left\{|Z|: Z \in \mathcal{Z}_{0}, x+Z \subset K\right\} & \text { if } x \in \operatorname{int}(K) \\
0 & \text { if } x \notin \operatorname{int}(K) .
\end{array}\right.
$$

It is clear that $K \rightarrow g_{K}$ is continuous from $\mathcal{K}_{n}$ to $C_{0}\left(\mathbb{R}^{n}\right)$. Since convex combinations (for the Minkowski addition) of zonoids are zonoids, it follows as in Proposition 11, that $g_{K}$ reaches its maximum at a unique point $z(K)$ and that $g_{K}^{1 / n}$ is concave on its support. Thus

$$
K_{z}(c)=\left\{x \in \mathbb{R}^{n}: g_{K}(x) \geq c\left\|g_{K}\right\|_{\infty}\right\}, \quad 0 \leq c \leq 1,
$$

is convex. And, again with Lemma 15, we get the following proposition.

Proposition 13. The mapping $z: \mathcal{K}_{n} \rightarrow \mathbb{R}^{n}$ is a proper affine invariant point and, for every $0<c \leq 1, K \rightarrow K_{z}(c)$ is a proper affine invariant set mapping.

Remark 9. (i) For $K \in \mathcal{K}_{n}$ and $x \in \operatorname{int}(K)$, define

$$
\phi_{K}(x)=\max \{\lambda>0: x-\lambda(K-g(K)) \subset K-g(K)\},
$$

where $g(K)$ is the centroid of $K$. Then $\phi_{K}$ is positive and concave on $\operatorname{int}(K)$ and $\phi_{K}(x) \rightarrow 0$ when $x \rightarrow \partial K$. So one can extend $\phi_{K}$ to a continuous function on $\mathbb{R}^{n}$ by setting $\phi_{K}(x)=0$ when $x \notin \operatorname{int}(K)$. It is well known that $\max _{x} \phi_{K}(x) \geq \phi_{K}(g(K)) \geq \frac{1}{n}$. It follows easily that for any $0<\delta<1$,

$$
K \rightarrow\left\{\phi_{K} \geq(1-\delta) \max \phi_{K}\right\}
$$

is an affine invariant set mapping.

But generally, $\phi_{K}$ does not reach its maximum at a unique point. To see that, take $K=\Delta_{2} \times[-1,1]^{n-2} \subset \mathbb{R}^{n}$, where $\Delta_{2}$ is a regular simplex centered at 0 in $\mathbb{R}^{2}$. Then

$$
\left\{x \in \mathbb{R}^{n}: \phi_{K}(x)=\max \phi_{K}\right\}=\left\{\left(0,0, x_{3}, \ldots, x_{n}\right):\left|x_{i}\right| \leq \frac{1}{2}, 3 \leq i \leq n\right\} .
$$


(ii) Instead of the centers of centrally symmetric convex bodies contained in $K$, we may study the centers of those containing $K$. For $K \in \mathcal{K}_{n}$, we define a positive and convex function $\rho_{K}: \mathbb{R}^{n} \rightarrow \mathbb{R}_{+}$by

$$
\rho_{K}(x)=|\operatorname{conv}[K, 2 x-K]| .
$$

If $L \in \mathcal{K}_{n}$ is centered at $x \in \mathbb{R}^{n}$ and satisfies $K \subseteq L$, then $2 x-K \subseteq L$ and therefore $\operatorname{conv}(K, 2 x-K) \subseteq L$. It follows that

$$
\min \left\{|L|: L \in \mathcal{K}_{n}, K \subseteq L, L \text { is centrally symmetric }\right\}=\min _{x} \rho_{K}(x) .
$$

It is clear that $\rho_{K} \rightarrow+\infty$, when $|x| \rightarrow \infty$. For $c>1$, let

$$
N_{c}(K)=\left\{x \in \mathbb{R}^{n}: \rho_{K}(x) \leq c \min _{z \in \mathbb{R}^{n}} \rho_{K}(z)\right\} .
$$

Then $K \rightarrow N_{c}(K)$ is an affine invariant set mapping.

However, one cannot define an affine invariant point in that way, because it may happen that $\rho_{K}$ does not reach its minimum at a unique point. For instance, if $K$ is a simplex in $\mathbb{R}^{n}$ and $n$ is even, then $\left\{\rho_{K}=\min \rho_{K}\right\}$ has non-empty interior.

\section{The illumination body.}

Let $K \in \mathcal{K}_{n}, \delta \geq 0, x \in \mathbb{R}^{n}$ and $F_{K}(x)=|\operatorname{conv}(x, K)|$. The illumination body $K^{\delta}$ [24] of $K$ is defined by

$$
K^{\delta}=\left\{x \in \mathbb{R}^{n}: F_{K}(x) \leq(1+\delta)|K|\right\} .
$$

Then $K^{\delta} \in \mathcal{K}_{n}$. Clearly $K^{0}=K \subseteq K^{\delta}$ and $K \rightarrow K^{\delta}$ is affine invariant.

If $\sigma_{K}$ is the surface measure of $K$ on $S^{n-1}$ and $h_{K}: \mathbb{R}^{n} \rightarrow \mathbb{R}, h_{K}(\xi)=\sup _{x \in K}\langle\xi, x\rangle$, is the support function of $K$, then

$$
F_{K}(x)=\frac{1}{n} \int_{S^{n-1}} \max \left(\langle x, u\rangle, h_{K}(u)\right) d \sigma_{K}(u) .
$$

Thus $F_{K}$ is continuous, convex and clearly $F_{K}(x) \rightarrow+\infty$, when $|x| \rightarrow+\infty$.

Proposition 14. Let $I^{\delta}: \mathcal{K}_{n} \rightarrow \mathcal{K}_{n}$ be defined by $I^{\delta}(K)=K^{\delta}$. Then $I^{\delta} \in \mathfrak{A}_{n}$.

Proof. We prove the continuity. Fix $\delta>0$ and $K \in \mathcal{K}_{n}$. We may suppose that $g(K)=0$, and that for some $0<r \leq R<\infty, r B_{2}^{n} \subseteq K \subseteq R B_{2}^{n}$. For $0<\eta<1$, there exists $\varepsilon>0$, such that for all $L \in \mathcal{K}_{n}$ with $d_{H}(K, L) \leq \varepsilon$, one has, $(1-\eta) K \subseteq L \subset(1+\eta) K$. Thus, for $x \in \mathbb{R}^{n}$,

$$
\begin{aligned}
(1-\eta) \operatorname{conv}[x, K] & \subset \operatorname{conv}[(1-\eta) x,(1-\eta) K] \subseteq \operatorname{conv}[x,(1-\eta) K] \subset \operatorname{conv}[x, L] \\
& \subset \operatorname{conv}[x,(1+\eta) K] \subset \operatorname{conv}[(1+\eta) x,(1+\eta) K] \\
& =(1+\eta) \operatorname{conv}[x, K]
\end{aligned}
$$

It follows that for $t>0$,

$$
\left\{x \in \mathbb{R}^{n}: F_{K} \leq \frac{t}{(1+\eta)^{n}}\right\} \subseteq\left\{x \in \mathbb{R}^{n}: F_{L} \leq t\right\} \subseteq\left\{x \in \mathbb{R}^{n}: F_{K} \leq \frac{t}{(1-\eta)^{n}}\right\} .
$$


Moreover, ||$K|-| L|| \leq \rho$, where $\rho=2 n \eta \max \{|K|,|L|\}$. Consequently,

$$
\left\{x \in \mathbb{R}^{n}: F_{K} \leq \frac{(1+\delta)(1-\rho)}{(1+\eta)^{n}}|K|\right\} \subseteq L^{\delta} \subseteq\left\{x \in \mathbb{R}^{n}: F_{K} \leq \frac{(1+\delta)(1+\rho)}{(1-\eta)^{n}}|K|\right\} .
$$

This allows to conclude, because $\delta \rightarrow\left\{F_{K} \leq(1+\delta)|K|\right\}$ is continuous.

\section{The convex floating body.}

For a convex body $K$ and for $0 \leq \delta<\left(\frac{n}{n+1}\right)^{n}$, the convex floating body $K_{\delta}$ of $K$ was defined in 20] as the intersection of all halfspaces $H^{+}$whose defining hyperplanes $H$ cut off a set of volume at most $\delta|K|$ from $K$,

$$
K_{\delta}=\bigcap_{\left|H^{-} \cap K\right| \leq \delta|K|} H^{+} .
$$

Then the map $F_{\delta}: \mathcal{K}_{n} \rightarrow \mathcal{K}_{n}$, defined by $F_{\delta}(K)=K_{\delta}$ is an affine invariant set mapping. This, and affine invariant points defined via the convex floating body are treated in [13.

\section{Extension to subsets of $\mathbb{R}^{n}$ of affine invariant points on $\mathcal{K}_{k}, 1 \leq k \leq n-1$.}

Let $\mathcal{K}_{n, k}$ be the set of closed convex subsets $L$ of $\mathbb{R}^{n}$, whose affine span $E_{L}$ is $k$ dimensional. If $L \in \mathcal{K}_{n, k}$, there exists a (non unique) one-to-one affine map $U: \mathbb{R}^{n}=$ $\mathbb{R}^{k} \times \mathbb{R}^{n-k} \rightarrow \mathbb{R}^{n}$ such that $U\left(\mathbb{R}^{k}\right)=E_{L}$. For $p \in \mathfrak{P}_{k}$, we then define

$$
p(L)=U\left(p\left(U^{-1}(L)\right)\right) .
$$

It is easy to show that this definition does not depend of the choice of $U$. Moreover this extended $p$ satisfies $p(L) \in E_{L}$ for every $L \in \mathcal{K}_{n, k}$ and it is affine invariant: For any one-to-one affine mapping $W: \mathbb{R}^{n} \rightarrow \mathbb{R}^{n}$, one has

$$
p((W(L))=W(p(L)) .
$$

Definition 8. We then call $p: \mathcal{K}_{n, k} \rightarrow \mathbb{R}^{n}$ defined by [16), an extended affine invariant point.

Example 1. For $k=1$, the unique invariant point is the midpoint of a segment. It is canonically extended to be the midpoint of any segment of $\mathbb{R}^{n}$. Another natural example is the centroid of a body in $\mathbb{R}^{k}$, which extends to the centroid of $L$ in $E_{L}$, when $L \in \mathcal{K}_{n, k}$.

The following proposition summarizes all these facts.

Proposition 15. Let $p: \mathcal{K}_{n, k} \rightarrow \mathbb{R}^{n}$ be the affine invariant extension of $p: \mathcal{K}_{k} \rightarrow \mathbb{R}^{k}$. Then $p(L) \in E_{L}$ for any $L \in \mathcal{K}_{n, k} . p$ is continuous, when $\mathcal{K}_{n, k}$ is endowed with the Hausdorff metric. Moreover, this extension is proper (in the sense that $p(L)$ is in the relative interior of $L$ in $\left.E_{L}\right)$, whenever $p: \mathcal{K}_{k} \rightarrow \mathbb{R}^{k}$ is proper.

Let $\mathcal{M}_{+}\left(\mathbb{R}^{n}\right)$ be the cone of non-negative Radon measures on $\mathbb{R}^{n}$. For a Borel function $f: \mathbb{R}^{n} \rightarrow \mathbb{R}^{n}$ and $\mu \in \mathcal{M}_{+}\left(\mathbb{R}^{n}\right)$, let $f(\mu)$ be the image measure of $\mu$ by $f$, i.e.

$$
(f(\mu))(B)=\mu\left(f^{-1}(B)\right) \text {, for any Borel subset } B \text { of } \mathbb{R}^{n} .
$$

For a map $M: \mathcal{K}_{n} \rightarrow \mathcal{M}_{+}\left(\mathbb{R}^{n}\right)$, denote $\mu_{K}=M(K)$. 
Definition 9. We say that a mapping $M: \mathcal{K}_{n} \rightarrow \mathcal{M}_{+}\left(\mathbb{R}^{n}\right)$ is a an affine invariant measure map if it is continuous for the Hausdorff topology on $\mathcal{K}_{n}$ and the weak ${ }^{*}$-topology on $\mathcal{M}_{+}\left(\mathbb{R}^{n}\right)$ and if $\mu_{U K}=U\left(\mu_{K}\right)$ for any affine one-to-one mapping $U: \mathbb{R}^{n} \rightarrow \mathbb{R}^{n}$. Thus

$$
\int h(y) d \mu_{U K}(y)=\int h(U x) d \mu_{K}(x), \text { for any non-negative Borel function } h \text { on } \mathbb{R}^{n} .
$$

Example 2. (i) Let $A \in \mathfrak{A}_{n}$. Let $\mu_{K}=\frac{\mathbf{1}_{A(K)}}{|A(K)|} d x$. Then for every non negative Borel function $h$,

$$
\int h(x) d \mu_{K}(x)=\frac{1}{|A(K)|} \int_{A(K)} h(x) d x .
$$

If $U: \mathbb{R}^{n} \rightarrow \mathbb{R}^{n}$ is an affine one-to-one mapping, then

$$
\begin{aligned}
\int h(y) d \mu_{U K}(y) & =\frac{1}{|A(U K)|} \int_{A(U K)} h(y) d y=\frac{1}{\mid(U(A(K)) \mid} \int_{(U(A(K))} h(y) d y \\
& =\frac{1}{|\operatorname{det}(U)||A(K)|} \int_{A(K)}|\operatorname{det}(U)| h(U x) d x=\int h(U x) d \mu_{K}(x) .
\end{aligned}
$$

(ii) Let $K \in \mathcal{K}_{n}$ and let $p \in \mathfrak{P}_{n}$ be proper. For $x \in \mathbb{R}^{n}$, let

$$
\|x-p(K)\|_{K-p(K)}=\inf \{\lambda \geq 0: x-p(K) \in \lambda(K-p(K))\} .
$$

If $\phi: \mathbb{R}_{+} \rightarrow \mathbb{R}_{+}$is a Borel function, let

$$
\mu_{K}=\frac{1}{|K|} \phi\left(\|x-p(K)\|_{K-p(K)}\right) d x
$$

Then, as above, it is easy to see that $K \rightarrow \mu_{K}$ is an affine invariant measure map.

(iii) Let $a$ and $\delta$ be strictly positive real numbers. We take $\phi(t)=\frac{1}{\delta} \mathbf{1}_{[a, a+\delta)}$ in (17) of Example (ii). Let $L=K-g(K)$. Then for any Borel function $h$, when $\delta \rightarrow 0$,

$$
\begin{aligned}
\int h(x) d \mu_{K}(x) & =\frac{1}{\delta|L|} \int_{a \leq\|x-g(K)\|_{L} \leq a+\delta} h(x) d x \\
& =\frac{n\left|B_{2}^{n}\right|}{\delta|L|} \int_{S^{n-1}}\left(\int_{\frac{a}{\|\theta\|_{L}} \leq r \leq \frac{a+\delta}{\|\theta\|_{L}}} h(r \theta) r^{n-1} d r\right) d \sigma(\theta) \\
& \sim \frac{v_{n}}{|L|} \int_{S^{n-1}} h\left(\frac{a \theta}{\|\theta\|_{L}}\right) \frac{1}{\|\theta\|_{L}^{n}} d \sigma(\theta) .
\end{aligned}
$$

Here, $\sigma$ is the normalized measure on $S^{n-1}$. Therefore, $K \rightarrow \mu_{K}$ is an affine invariant measure map, with $\mu_{K}$ given by

$$
\int h(x) d \mu_{K}(x)=\frac{1}{|L|} \int_{S^{n-1}} h\left(\frac{a \theta}{\|\theta\|_{L}}\right) \frac{1}{\|\theta\|_{L}^{n}} d \sigma(\theta) .
$$

With the previous definition, the following result is easy to prove. 
Proposition 16. Let $p \in \mathfrak{P}_{n}$, let $q: \mathcal{K}_{n, n-1} \rightarrow \mathbb{R}^{n}$ be an extended affine invariant point and let $\mu$ be an affine invariant measure such that $\mu_{K}=\mu(K)$ is supported by $K$ for all $K \in \mathcal{K}_{n}$. Then the map $d: \mathcal{K}_{n} \rightarrow \mathbb{R}^{n}$ defined by

$$
\left.K \rightarrow d(K)=p(K)+\int q\left(\left\{x \in K-p(K):\left\langle x^{*}, x\right\rangle=1\right\}\right) d \mu_{(K-p(K))}\right)^{\circ}\left(x^{*}\right),
$$

is an affine invariant point.

Example 3. For $0<\alpha<1$ and $w \in \mathfrak{P}_{n}$, we define $d_{1}$ and $d_{2} \in \mathfrak{P}_{n}$ by

$$
\begin{aligned}
& d_{1}(K)=p(K)+\frac{1}{\left|(K-p(K))^{\circ}\right|} \int_{S^{n-1}} \frac{q\left(\left\{x \in K-p(K):\langle\theta, x\rangle=\alpha\|\theta\|_{\left.\left.(K-p(K))^{\circ}\right\}\right)}\right.\right.}{\|\theta\|_{(K-p(K))^{\circ}}^{n}} d \sigma(\theta) . \\
& d_{2}(K)=p(K)+\frac{1}{\left|(K-p(K))^{\circ}\right|} \int_{S^{n-1}} \frac{w\left(\left\{x \in K-p(K):\langle\theta, x\rangle \geq \alpha\|\theta\|_{\left.\left.(K-p(K))^{\circ}\right\}\right)}\right.\right.}{\|\theta\|_{(K-p(K))^{\circ}}^{n}} d \sigma(\theta) .
\end{aligned}
$$

\section{Two affine invariant points related to the projection body.}

Let $K \in \mathcal{K}_{n}$. We recall that the support function $h_{\Pi K}: \mathbb{R}^{n} \rightarrow \mathbb{R}_{+}$of the projection body $\Pi K$ of $K$ (see e.g., [1, 18]) is given by

$$
h_{\Pi K}(x)=\left\{\begin{array}{cc}
|x| P_{u_{x}} K \mid & \text { if } x \neq 0 \\
0 & \text { if } x=0,
\end{array}\right.
$$

where $u_{x}=\frac{x}{|x|} \in S^{n-1}$ and for $u \in S^{n-1}, P_{u}: \mathbb{R}^{n} \rightarrow \mathbb{R}^{n}$ is the orthogonal projection onto $\{u\}^{\perp}$. It is well known (see [1, 18) that for any one-to-one linear map $T: \mathbb{R}^{n} \rightarrow \mathbb{R}^{n}$, one has

$$
h_{\Pi(T K)}(T x)=|\operatorname{det}(T)| h_{\Pi K}(x) \text { for all } x \in \mathbb{R}^{n} .
$$

We define two affine invariant points related to the projection body. In both definitions we use the centroid $g$ which could be replaced by any other $p \in \mathfrak{P}_{n}$.

(i) For $K \in \mathcal{K}_{n}$, let $\pi(K)=\frac{1}{|K|^{2}} \int_{K-g(K)} x h_{\Pi K}(x) d x+g(K)$. Then $\pi: \mathcal{K}_{n} \rightarrow \mathbb{R}^{n}$ is an affine invariant point. Observe that

$$
\pi(K)-g(K)=\frac{1}{|K|^{2}} \int_{K-g(K)} x h_{\Pi K}(x) d x=\frac{1}{(n+2)|K|^{2}} \int_{S^{n-1}} \frac{\theta}{\|\theta\|_{K-g(K)}^{n+2}} h_{\Pi K}(\theta) d \sigma(\theta) .
$$

(ii) For $K \in \mathcal{K}_{n}$, let $b_{t}(K)=\frac{1}{|K|} \int_{K-g(K)} g(K+t[-x, x]) d x$.

\section{References}

[1] R.J. Gardner Geometric tomography. Second edition. Encyclopedia of Mathematics and its Applications, 58. Cambridge University Press, Cambridge (2006).

[2] R. J. Gardner, The dual Brunn-Minkowski theory for bounded Borel sets: Dual affine quermassintegrals and inequalities, Adv. Math. 216 (2007), 358-386. 
[3] B. Grünbaum, Measures of symmetry for convex sets, Proc. Sympos. Pure Math. 7, (1963), 233-270.

[4] C. Haberl, Blaschke valuations, Amer. J. Math., 133, (2011), 717-751.

[5] M. Ludwig, Ellipsoids and matrix valued valuations, Duke Math. J. 119 (2003), 159-188.

[6] M. Ludwig, Minkowski areas and valuations, J. Differential Geometry, 86, (2010), 133-162.

[7] M. Ludwig And M. Reitzner, A classification of $S L(n)$ invariant valuations. Annals of Math. 172 (2010), 1223-1271.

[8] E. Lutwak, The Brunn-Minkowski-Firey theory II : Affine and geominimal surface areas, Adv. Math. 118 (1996), 244-294.

[9] E. Lutwak, D. Yang And G. Zhang, The Cramer-Rao inequality for star bodies, Duke Math. J. 112 (2002), 59-81.

[10] E. Lutwak, D. Yang And G. Zhang, Volume inequalities for subspaces of $L_{p}$, J. Differential Geom. 68 (2004), 159-184.

[11] M. Meyer And E. Werner, The Santalo-regions of a convex body, Transactions of the AMS 350 (1998), 4569-4591.

[12] M. Meyer, C. Schütt And E. Werner, New affine measures of symmetry for convex bodies, Adv. Math. 228, (2011), 2920-2942.

[13] M. Meyer, C. Schütt And E. Werner, Affine invariant points, Preprint 2012

[14] F. Nazarov, F. Petrov, D. Ryabogin and A. Zvavitch, A remark on the Mahler conjecture: local minimality of the unit cube, Duke Mathematical J. 154, (2010), 419-430.

[15] Relative entropy of cone measures and $L_{p}$ Centroid Bodies Proc. London Math. Soc. (3) 104 (2012), no. 2, 253-286.

[16] W. Rudin, Functional Analysis, McGraw-Hill, 1991.

[17] L. A. Santaló, Un invariante afin para los cuerpos convexos del espacio de $n$ dimensiones, Portugal. Math. 8 (1949), 155-161.

[18] R. SchneIder, Convex Bodies: The Brunn-Minkowski Theory, Encyclopedia of Mathematics and its Applications 44, Cambridge University Press, Cambridge (1993).

[19] F. Schuster, Crofton measures and Minkowski valuations, Duke Math. J. 154 (2010), $1-30$.

[20] C. Schütt And E. Werner, The convex floating body, Math. Scand. 66, (1990), 275-290.

[21] C. Schütt And E. Werner, Surface bodies and p-affine surface area. Adv. Math. 187 (2004), 98-145.

[22] A. Stancu, On the number of solutions to the discrete two-dimensional $L_{0}$-Minkowski problem. Adv. Math. 180 (2003), 290-323.

[23] N. Tomczak Jaegerman, Banach-Mazur Distances and Finite-Dimensional Operator Ideals, Encyclopedia of Mathematics and its Applications 44, Cambridge University Press, Cambridge (1993).

[24] E. WERNER, Illumination bodies and affine surface area, Studia Math. 110 (1994), 257269. 
[25] E. Werner, Rényi Divergence and $L_{p}$-affine surface area for convex bodies, Advances in Mathematics 230, (2012), 1040-1059

[26] E. Werner And D. Ye, New $L_{p}$ affine isoperimetric inequalities, Adv. Math. 218 (2008), no. $3,762-780$.

[27] E. Werner AND D. YE, Inequalities for mixed p-affine surface area, Math. Ann. 347 (2010), 703-737.

Mathieu Meyer

Université de Paris Est - Marne-la-Vallée

Equipe d'Analyse et de Mathématiques Appliquées

Cité Descartes - 5, bd Descartes

Champs-sur-Marne 77454 Marne-la-Vallée, France

mathieu.meyer@univ-mlv.fr

Carsten Schütt

Christian Albrechts Universität

Mathematisches Seminar

24098 Kiel, Germany

schuett@math.uni-kiel.de

Elisabeth Werner

Department of Mathematics

Case Western Reserve University

Université de Lille 1

Cleveland, Ohio 44106, U. S. A.

elisabeth.werner@case.edu

UFR de Mathématiques

59655 Villeneuve d'Ascq, France 